\title{
THE GRASSMANN ALGEBRA OVER ARBITRARY RINGS AND MINUS SIGN IN ARBITRARY CHARACTERISTIC
}

\author{
GAL DOR, ALEXEI KANEL-BELOV, AND UZI VISHNE
}

\begin{abstract}
An analog in characteristic 2 for the Grassmann algebra $G$ was essential in a counterexample to the long standing Specht conjecture. We define a generalization $\mathfrak{G}$ of the Grassmann algebra, which is well-behaved over arbitrary commutative rings $C$, even when 2 is not invertible. This lays the foundation for a supertheory over arbitrary base ring, allowing one to consider general deformations of superalgebras.

The construction is based on a generalized sign function. It enables us to provide a basis of the non-graded multilinear identities of the free superalgebra with supertrace, valid over any ring.

We also show that all identities of $\mathfrak{G}$ follow from the Grassmann identity, and explicitly give its co-modules, which turn out to be generalizations of the sign representation. In particular, we show that the $n$th co-module is a free $C$-module of rank $2^{n-1}$.
\end{abstract}

\section{The Specht problem, sign, and Grassmann algebra}

Supertheory, based on the partition of objects into even and odd parts, is ubiquitous in mathematics. It features the Grassmann algebra, whose definition requires the sign, and thus avoids characteristic 2, hampering the development of a complete supertheory. Indeed, in order to allow arbitrary reductions and quotients, it is important to consider such objects over arbitrary base rings and fields, in arbitrary characteristic. Similarly, one would like to investigate noncommutativity under deformations of the base ring, similarly to Poisson brackets in quantum mechanics. Our starting point is a Grassmann algebra in characteristic 2, which was constructed in connection with the famous Specht problem. We present a characteristic-free construction leading to generalized notions of superalgebras and superidentities, valid over an arbitrary commutative ring, thus supporting the possibility of having a supertheory in arbitrary characteristic.

Let $C$ be an arbitrary commutative unital ring, and let $C\langle X\rangle$ be the free (associative) algebra over a countable infinite alphabet $X$. Recall that an ideal of $C\langle X\rangle$ is a T-ideal if it is closed under transformations.

Received by the editors July 9, 2015, and, in revised form, February 27, 2018, and August 7, 2019 .

2010 Mathematics Subject Classification. Primary 16R10; Secondary 17A70, 16R30, 16R50.

Key words and phrases. Superalgebra, generalized Grassmann algebra, generalized sign, polynomial identities, trace identities.

This work was supported by BSF grant 2010/149, ISF grants 1207/12 and 1994/20, and RSF grant 17-11-01377.

(C) 2020 by the authors under Creative Commons Attribution-Noncommercial 3.0 License (CC BY NC 3.0) 
The Specht problem asks whether T-ideals are always finitely based, namely generated as T-ideals by some finite set. The Specht problem has been answered negatively for the analogous cases of groups and Lie algebras, which made the following result by Kemer [Kem91, Theorem 2.4] quite surprising.

Theorem 1.1 (Specht Property for algebras over fields). Let $A$ be an (associative) algebra over a field $C=\mathbb{F}$ of characteristic zero. Then the T-ideal of identities $\operatorname{id}(A)$ is finitely based.

This positive answer to the Specht problem in characteristic zero does not extend well to other characteristics, and has in fact been disproved for all non-zero characteristics. Additionally, there is no known method of actually finding the finite basis of the identities of a given algebra, and in fact, there are only a few natural cases where a complete basis of identities is known.

Kemer proved his theorem via a series of reductions, first to the case of the Tideal of identities of an affine algebra, and then it was shown that any T-ideal of identities of an affine algebra is also the T-ideal of identities of a finite-dimensional algebra.

One concept of vital importance in the proof of Theorem 1.1 is the Grassmann algebra. The Grassmann algebra $G$ over a field $\mathbb{F}$ where char $\mathbb{F} \neq 2$ is the algebra generated by a countable set of generators $e_{1}, e_{2}, \ldots$ under the relations:

$$
e_{i} e_{j}=-e_{j} e_{i} \text {. }
$$

Remark 1.2. PI-theory in characteristic zero has quite a lot of information on $G$. For instance, it is known that when $\mathbb{F}$ is infinite, $\operatorname{id}(G)$ is generated by the Grassmann identity $[x,[y, z]]=0$.

The structure of $G$ is related to the notion of a superalgebra: an algebra $A=$ $A_{0} \oplus A_{1}$ satisfying $A_{0} A_{0} \subseteq A_{0}, A_{1} A_{1} \subseteq A_{0}, A_{0} A_{1} \subseteq A_{1}$ and $A_{1} A_{0} \subseteq A_{1}$. The subalgebra $A_{0}$ is its even part, and the $A_{0}$-module $A_{1}$ is its odd part. Additionally, the decomposition $A=A_{0} \oplus A_{1}$ is referred to as the ( $\mathbb{Z} / 2 \mathbb{Z}$-) grading of $A$. When we refer to a superalgebra $A$, we are actually referring to a specific grading $A=A_{0} \oplus A_{1}$, because in general there are many possible such gradings. An element $x$ in $A_{0}$ or $A_{1}$ is called homogenous, and we let $|x|=0$ if $x \in A_{0}$ and $|x|=1$ if $x \in A_{1}$.

Indeed, $G$ is a superalgebra when we take $G_{0}$ to be the space spanned by all words of even length in the generators $e_{1}, e_{2}, \ldots$ and $G_{1}$ the space spanned by words of odd length.

In general, when $A=A_{0} \oplus A_{1}$ is a superalgebra, the supercommutator of homogeneous elements $x, y \in A$ is given by

$$
\{x, y\}=x y-(-1)^{|x| \cdot|y|} y x .
$$

If the supercommutator of $x$ and $y$ is zero for all $x, y \in A$, then we say that $A$ is supercommutative. Then with respect to the grading defined above, $G$ becomes supercommutative.

One defines the free supercommutative algebra $S$ over $C$ as the superalgebra generated by countably many even generators $y_{1}, y_{2}, y_{3}, \ldots$ and countably many odd generators $z_{1}, z_{2}, z_{3}, \ldots$ whose only relations are $\left\{x_{1}, x_{2}\right\}=0$ for every $x_{1}, x_{2} \in$ $S$. Note that $S \cong C\left[y_{1}, \ldots\right] \otimes_{C} G$ as superalgebras, with the isomorphism given by $y_{i} \mapsto y_{i} \otimes 1$ and $z_{i} \mapsto 1 \otimes e_{i}$. In particular when $C$ is an infinite field, $\operatorname{id}(S)=\operatorname{id}(G)$.

One can build a theory of super linear algebra, with supertraces, superdeterminants (also known as Berezians) etc. (see DM99, KT94). We merely note that 
the basic axiom of traces, $\operatorname{tr}[a, b]=0$, becomes $\operatorname{str}\{a, b\}=0$ in the case of the supertrace. So, for example,

Definition 1.3. If $A$ is any algebra with trace tr, then the algebra $A \otimes G$ inherits the grading of $G$, and the function $\operatorname{str}(a \otimes w)=\operatorname{tr}(a) \otimes w$ becomes a supertrace. We will refer to this as the supertrace associated with $A \otimes G$.

Remark 1.4. The supertrace associated with $A \otimes G$ is indeed a supertrace because of the easily verified fact that

$$
\{a \otimes w, b \otimes u\}=[a, b] \otimes w u,
$$

for all $a, b \in A$ and $u, w \in G$.

In other words, tensoring by $G$ turns algebras into superalgebras, commutators into supercommutators, and traces into supertraces. The role of $G$ and superalgebras in general in PI-theory is best illustrated by the following deep theorem of Kemer, which reduces the study of arbitrary PI-algebras in characteristic 0 to the study of finite-dimensional PI-superalgebras.

Theorem 1.5 (Kemer's Superrepresentability Theorem). For any algebra A over a field of characteristic 0 , there is some finite-dimensional superalgebra $B$ such that $\operatorname{id}(A)=\operatorname{id}(G[B])$, where $G[B]=\left(G_{0} \otimes B_{0}\right) \oplus\left(G_{1} \otimes B_{1}\right)$ is the Grassmann hull of $B$.

The main problem with $G$ is that it cannot be easily generalized to arbitrary characteristics. In particular, in characteristic 2 the relation (11) implies that the algebra is commutative. For this reason, Bel00 came up with the following algebra, which was the basis for Belov's counterexample to the Specht problem in characteristic 2 (see [BR05, p. 204] for details):

Definition 1.6. Define the extended Grassmann algebra $G^{+}$over a field $\mathbb{F}$ of characteristic 2 as the algebra generated by elements $\varepsilon_{1}, \varepsilon_{2}, \ldots$ and $e_{1}, e_{2}, \ldots$, such that the $\varepsilon_{i}$ are central,

$$
\varepsilon_{i}^{2}=0
$$

and the following relation is satisfied:

$$
\left[e_{i}, e_{j}\right]=\varepsilon_{i} \varepsilon_{j} e_{i} e_{j}
$$

So, in fact, $G^{+}$is an algebra over the local algebra $\mathbb{F}\left[\varepsilon_{1}, \varepsilon_{2}, \ldots\right]$.

This algebra was used to produce counterexamples in characteristic 2, such as constructing a T-ideal that is not finitely based (see for example BR05. p. 210, Example 7.22]), as well as to investigate the T-space structure of the relatively free algebra generated by the Grassmann identity [GTS11, GT09, Tsy09.

Remark 1.7. The ideal of identities $\operatorname{id}\left(G^{+}\right)$is generated by the same identity as the Grassmann algebra, namely $[x,[y, z]]=0$ (see Remark [1.2) .

The main disadvantage of $G^{+}$is that it degenerates in any characteristic not equal to 2 , and superficially looks very different from the ordinary Grassmann algebra $G$. Therefore, our goal in this paper is to present and study an algebra $\mathfrak{G}$, unifying both constructions in a way that is well-behaved over arbitrary commutative rings. We show that $\mathfrak{G}$ possesses properties similar to the ordinary Grassmann algebra $G$, and generalize various theorems regarding $G$ over fields of characteristics not 2 to theorems on $\mathfrak{G}$ which hold in general. 
In particular, we prove that $\mathrm{id}(\mathfrak{G})$ is generated as a T-ideal by the Grassmann identity, $[x,[y, z]]=0$ (Theorem [3.5). Moreover, when 2 is invertible in $C, \mathfrak{G}$ is strongly PI-equivalent to the free supercommutative algebra $S$, in the sense that $\operatorname{id}\left(A \otimes_{C} S\right)=\operatorname{id}\left(A \otimes_{C} \mathfrak{G}\right)$ for every $C$-algebra $A$ (Theorem 3.14).

Next, we present a generalization of the notion of signs of permutations, that is associated with $\mathfrak{G}$ in much the same way that ordinary signs are associated with the ordinary Grassmann algebra $G$. We refer to this generalization as the generalized sign representation. We show that over any commutative $\operatorname{ring} C$, the generalized sign representation is actually the full co-module of $\mathfrak{G}$ : The $S_{n}$-module of generalized signs $C[\varepsilon]_{n}$ over a ring $C$ is the $n$-th co-module of $\mathfrak{G}$ (Theorem 3.28). Furthermore, the $n$-th co-module of $\mathfrak{G}$ is a free $C$-module, of rank $2^{n-1}$ (Theorem 3.31). This generalizes the well known fact that the co-dimension sequence of $G$ (in characteristic not 2) is $c_{n}(G)=2^{n-1}$.

The ring $C[\varepsilon]$ is defined in Subsection 3.1. In Section 4 we define generalized superalgebras (henceforth called $\Sigma$-superalgebras for brevity), as algebras over $C[\varepsilon]$ which are graded by the group $(\mathbb{Z} / 2 \mathbb{Z})^{\mathbb{N}}$. The free $\Sigma$-superalgebra $\mathfrak{S}$ is defined in Example 4.5. Using this object we define the generalized Grassmann hull of an arbitrary $\Sigma$-superalgebra $\mathfrak{A}$, and determine its $\Sigma$-superidentities in terms of the $\Sigma$-superidentities of $\mathfrak{A}$ (Theorem 4.14).

For the reader's convenience, let us collect here the notation used for the four objects studied and compared in this paper:

\begin{tabular}{c|cc} 
& superalgebra & $\Sigma$-superalgebra \\
\hline Grassmann & $G$ & $\mathfrak{G}$ \\
free commutative & $S$ & $\mathfrak{S}$
\end{tabular}

In Section 5 we define generalized supertraces ( $\Sigma$-supertraces), and show that when 2 is invertible, these notions coincide with the notions of ordinary supertheory:

Theorem (Theorem 5.8). Suppose that 2 is invertible in $C$. Let $A$ be some $C$ algebra with trace tr. Let $\mathfrak{s t r}$ be the associated $\Sigma$-supertrace of $A \otimes_{C} \mathfrak{S}$, and associate a supertrace str to $A \otimes_{C} S$. Then the supertrace identities of $A \otimes_{C} S$ are the same as the $\Sigma$-supertrace identities of $A \otimes_{C} \mathfrak{S}$, with $\mathfrak{s} \mathfrak{T} \mathfrak{r}$ replaced by $\mathrm{sTr}$.

The next question is what properties do supertraces (and more generally, $\Sigma$ supertraces) satisfy. Thus we turn our attention to the question of ungraded identities satisfied by supertraces. In Theorem 5.12 we give a complete basis of identities for the multilinear part of the ideal of identities of the free $\Sigma$-superalgebra with $\Sigma$-supertrace (over any ring).

Another approach to superalgebras in characteristic 2, based on [Ven16], was recently presented in [Kau18].

\section{Preliminaries}

Throughout the paper, algebras are associative, but not necessarily unital. The base ring $C$ will always be commutative and unital. We will assume nothing about the characteristic of $C$, except where explicitly stated.

Let $A$ be an algebra over $C$, and let $C\langle X\rangle$ be the free (associative) algebra over a countable infinite alphabet $X$. A polynomial $f\left(x_{1}, \ldots, x_{n}\right) \in C\langle X\rangle$ is an identity of $A$ if for all substitutions $a_{1}, \ldots, a_{n} \in A$, we have that $f\left(a_{1}, \ldots, a_{n}\right)=0$. We let

$$
\operatorname{id}(A)=\{f \in C\langle X\rangle \mid f \text { is an identity of } \mathrm{A}\} .
$$


denote the ideal of identities of $A$. An algebra satisfying some non-zero identity with at least one invertible coefficient is called a PI-algebra.

Obviously, $\operatorname{id}(A)$ is an ideal of $C\langle X\rangle$, which is invariant under substitutions. For any ring $R$, a $T$-ideal is an ideal $I \triangleleft R$ such that $\tau(I) \subseteq I$ for every endomorphism $\tau$ of $R$. We will implicitly assume throughout that all T-ideals are T-ideals of $C\langle X\rangle$. With this terminology, id $(A)$ is a T-ideal for every algebra $A$.

Given that an algebra $A$ over an infinite field $C=\mathbb{F}$ satisfies an identity $f$, it is always possible to break $f$ down into its multi-homogenous components, by multiplying each variable by suitable scalars, and using a standard Vandermondetype argument. Furthermore, in characteristic 0, one can multilinearize any identity to an equivalent multilinear identity. Thus, in characteristic 0 over a field, any Tideal is generated by its multilinear part.

Because of this, one considers the spaces

$$
P_{n}=\left\{\sum_{\sigma \in S_{n}} \alpha_{\sigma} x_{\sigma(1)} x_{\sigma(2)} \cdots x_{\sigma(n)} \mid \alpha_{\sigma} \in C\right\}
$$

of multilinear polynomials in the variables $x_{1}, \ldots, x_{n}$. This space has the structure of an $S_{n}$-module by defining:

$$
\tau \cdot x_{\sigma(1)} x_{\sigma(2)} \cdots x_{\sigma(n)}=x_{\tau \sigma(1)} x_{\tau \sigma(2)} \cdots x_{\tau \sigma(n)} .
$$

With the above definition, $C\left[S_{n}\right] \cong P_{n}$ as $S_{n}$-modules, with an isomorphism given by $\sigma \mapsto x_{\sigma(1)} x_{\sigma(2)} \cdots x_{\sigma(n)}$.

The multilinear part of degree $n$ of a T-ideal $\Delta$ is given by $\Delta \cap P_{n}$, which is an $S_{n}$-submodule of $P_{n}$. The quotient $P_{n} /\left(\Delta \cap P_{n}\right)$ is called the $n$-th co-module of $\Delta$, and (in case $C=\mathbb{F}$ is a field) $c_{n}=\operatorname{dim} P_{n} /\left(\Delta \cap P_{n}\right)$ is the $n$-th co-dimension.

Remark 2.1. In addition to Remark 1.2, it is known that the co-dimension sequence of $G$ is exactly $c_{n}=2^{n-1}$. This result is obtained by first applying a combinatoric argument showing that the identity $[x,[y, z]]=0$ has enough consequences to reduce the co-dimension to be $c_{n} \leq 2^{n-1}$, and then using the representation theory of $S_{n}$ to show that it is bounded from below by the same quantity.

\section{The generalized Grassmann Algebra}

The standard Grassmann algebra $G$ is well behaved in characteristic not 2, while the generalized Grassmann algebra $G^{+}$is defined in characteristic 2. Our first objective is to combine the two objects into an algebra defined over an arbitrary (commutative) ring, in a way which is amenable to reductions and inverse limits.

3.1. The generalized Grassmann algebra. Starting from the relations $\left[e_{i}, e_{j}\right]=$ $\varepsilon_{i} \varepsilon_{j} e_{i} e_{j}$ of Definition 1.6. we immediately obtain $-\varepsilon_{i} \varepsilon_{j} e_{i} e_{j}=-\left[e_{i}, e_{j}\right]=\left[e_{j}, e_{i}\right]=$ $\varepsilon_{i} \varepsilon_{j} e_{j} e_{i}=\varepsilon_{i} \varepsilon_{j}\left(1-\varepsilon_{i} \varepsilon_{j}\right) e_{i} e_{j}$, which will be satisfied by requiring $-\varepsilon_{i} \varepsilon_{j}=$ $\varepsilon_{i} \varepsilon_{j}\left(1-\varepsilon_{i} \varepsilon_{j}\right)$, or equivalently,

$$
\varepsilon_{i}^{2} \varepsilon_{j}^{2}=2 \varepsilon_{i} \varepsilon_{j}
$$

This observation motivates the following definition.

Definition 3.1. We denote by $C[\varepsilon]$ the commutative ring $C[\varepsilon]=C\left[\theta, \varepsilon_{1}, \varepsilon_{2}, \ldots\right]$, subject to the relations

$$
\varepsilon_{i}^{2}=\theta \varepsilon_{i} .
$$

and

$$
\theta^{2}=2 \text {. }
$$


Definition 3.2. The generalized Grassmann algebra $\mathfrak{G}$ over $C$ is the unital algebra generated by elements $e_{1}, e_{2}, \ldots$ over the central subring $C[\varepsilon]=C\left[\theta, \varepsilon_{1}, \varepsilon_{2}, \ldots\right]$ defined above, subject to the relations

$$
\left[e_{i}, e_{j}\right]=\varepsilon_{i} \varepsilon_{j} e_{i} e_{j}
$$

for every $i, j$ (in particular $\theta \varepsilon_{i} e_{i}^{2}=\varepsilon_{i}^{2} e_{i}^{2}=0$ ).

The following version of (3) will be frequently used:

$$
e_{j} e_{i}=\left(1-\varepsilon_{i} \varepsilon_{j}\right) e_{i} e_{j} .
$$

Remark 3.3. The elements $e_{j}^{2}$ are central in $\mathfrak{G}$, as

$$
e_{j}^{2} e_{i}=\left(1-\varepsilon_{i} \varepsilon_{j}\right)^{2} e_{i} e_{j}^{2}=\left(1-2 \varepsilon_{i} \varepsilon_{j}+\varepsilon_{i}^{2} \varepsilon_{j}^{2}\right) e_{i} e_{j}^{2}=e_{i} e_{j}^{2} .
$$

Modulo $\theta$ we recover the extended Grassmann algebra. More precisely:

Remark 3.4. The quotient $\mathfrak{G} / \theta \mathfrak{G}$ is the extended Grassmann algebra $G^{+}$over $C / 2 C$.

The terminology attached to $\mathfrak{G}$ is justified by the following theorem.

Theorem 3.5. Let $\mathfrak{G}$ be the generalized Grassmann algebra defined over $C$. Then $\operatorname{id}(\mathfrak{G})$ is generated as a T-ideal by the Grassmann identity, $[x,[y, z]]=0$.

We first show that $[x,[y, z]]=0$ holds in $\mathfrak{G}$, and then that all other identities of $\mathfrak{G}$ are consequences of it.

Lemma 3.6. Let $e_{1}, e_{2}, \cdots \in \mathfrak{G}$ be the generators as in Definition 3.2. Then,

(1) $\left[e_{i},\left[e_{j}, e_{k}\right]\right]=0$ for all $i, j$ and $k$.

(2) $\left[e_{i}, e_{j}\right]\left[e_{m}, e_{k}\right]+\left[e_{j}, e_{k}\right]\left[e_{i}, e_{m}\right]=0$ for all $i, j, k$ and $m$.

Proof. By (3), we have:

$$
\begin{aligned}
{\left[e_{i},\left[e_{j}, e_{k}\right]\right] } & =\left[e_{i}, \varepsilon_{j} \varepsilon_{k} e_{j} e_{k}\right] \\
& =\varepsilon_{j} \varepsilon_{k}\left[e_{i}, e_{j} e_{k}\right] \\
& =\varepsilon_{j} \varepsilon_{k}\left(\left[e_{i}, e_{j}\right] e_{k}+e_{j}\left[e_{i}, e_{k}\right]\right) \\
& =\varepsilon_{j} \varepsilon_{k}\left(\varepsilon_{i} \varepsilon_{j} e_{i} e_{j} e_{k}+\varepsilon_{i} \varepsilon_{k} e_{j} e_{i} e_{k}\right) \\
& =\left(\varepsilon_{i} \varepsilon_{j}^{2} \varepsilon_{k}+\varepsilon_{i} \varepsilon_{j} \varepsilon_{k}^{2}-\varepsilon_{i}^{2} \varepsilon_{j}^{2} \varepsilon_{k}^{2}\right) e_{i} e_{j} e_{k} \\
& =\left(\theta+\theta-\theta^{3}\right) \varepsilon_{i} \varepsilon_{j} \varepsilon_{k} e_{i} e_{j} e_{k} \\
& =(2 \theta-2 \theta) \varepsilon_{i} \varepsilon_{j} \varepsilon_{k} e_{i} e_{j} e_{k}=0 .
\end{aligned}
$$

Similarly,

$$
\begin{aligned}
{\left[e_{i}, e_{j}\right]\left[e_{m}, e_{k}\right] } & +\left[e_{j}, e_{k}\right]\left[e_{i}, e_{m}\right]=\varepsilon_{i} \varepsilon_{j} \varepsilon_{m} \varepsilon_{k}\left(e_{i} e_{j} e_{m} e_{k}+e_{j} e_{k} e_{i} e_{m}\right) \\
& =\varepsilon_{i} \varepsilon_{j} \varepsilon_{m} \varepsilon_{k}\left(1+\left(1-\varepsilon_{i} \varepsilon_{k}\right)\left(1-\varepsilon_{j} \varepsilon_{i}\right)\left(1-\varepsilon_{k} \varepsilon_{m}\right)\right) e_{i} e_{j} e_{m} e_{k} \\
& =\varepsilon_{i} \varepsilon_{j} \varepsilon_{m} \varepsilon_{k}\left(1+\left(1-\theta^{2}\right)\left(1-\theta^{2}\right)\left(1-\theta^{2}\right)\right) e_{i} e_{j} e_{m} e_{k} \\
& =\varepsilon_{i} \varepsilon_{j} \varepsilon_{m} \varepsilon_{k}(1-1) e_{i} e_{j} e_{m} e_{k}=0 .
\end{aligned}
$$

More generally:

Lemma 3.7. We have $\left[e_{i}, e_{j}\right]\left[u, e_{k}\right]+\left[e_{j}, e_{k}\right]\left[e_{i}, u\right]=0$ for every element $u \in \mathfrak{G}$. 
Proof. It suffices to check the claim for monomials. Let $u=e_{\ell_{1}} \cdots e_{\ell_{n}}$. Then, we have:

$$
\begin{aligned}
& {\left[e_{i}, e_{j}\right]\left[u, e_{k}\right]+\left[e_{j}, e_{k}\right]\left[e_{i}, u\right]=} \\
& \sum_{m} e_{\ell_{1}} \cdots e_{\ell_{m-1}}\left(\left[e_{i}, e_{j}\right]\left[e_{\ell_{m}}, e_{k}\right]+\left[e_{j}, e_{k}\right]\left[e_{i}, e_{\ell_{m}}\right]\right) e_{\ell_{m+1}} \cdots e_{\ell_{n}}=0,
\end{aligned}
$$

by Lemma 3.6 .

Lemma 3.8. The Grassmann identity $[x,[y, z]]$ is an identity of $\mathfrak{G}$.

Proof. We wish to show that all commutators are central. Thus, it suffices to show that they commute with the $e_{i}$ 's. So, we must show that $\left[e_{i},\left[w_{1}, w_{2}\right]\right]=0$ where $w_{1}$ and $w_{2}$ are some words in the generators. If the lengths of both $w_{1}$ and $w_{2}$ are 1 , then we are done by Lemma 3.6.(11). Otherwise, assume without loss of generality that $w_{1}=e_{j} u$, and assume via induction that we already have that $\left[e_{i},[x, y]\right]=0$ for all $i$ and for all words $x, y$ such that $x$ is not longer than $u$, and $y$ is not longer than $w_{2}$. Then

$$
\begin{aligned}
{\left[e_{i},\left[e_{j} u, w_{2}\right]\right] } & =\left[e_{i}, e_{j}\left[u, w_{2}\right]\right]+\left[e_{i},\left[e_{j}, w_{2}\right] u\right] \\
& =\left[e_{i}, e_{j}\right]\left[u, w_{2}\right]+e_{j}\left[e_{i},\left[u, w_{2}\right]\right]+\left[e_{i},\left[e_{j}, w_{2}\right]\right] u+\left[e_{j}, w_{2}\right]\left[e_{i}, u\right] \\
& =\left[e_{i}, e_{j}\right]\left[u, w_{2}\right]+\left[e_{j}, w_{2}\right]\left[e_{i}, u\right] .
\end{aligned}
$$

We need to prove that this is zero. We will do so by induction. If $w_{2}=e_{k} v$, and if we assume that the expression is zero for all shorter words, then

$$
\begin{gathered}
{\left[e_{i}, e_{j}\right]\left[u, w_{2}\right]+\left[e_{j}, w_{2}\right]\left[e_{i}, u\right]=\left[e_{i}, e_{j}\right]\left[u, e_{k} v\right]+\left[e_{j}, e_{k} v\right]\left[e_{i}, u\right]=} \\
{\left[e_{i}, e_{j}\right]\left[u, e_{k}\right] v+\left[e_{i}, e_{j}\right] e_{k}[u, v]+e_{k}\left[e_{j}, v\right]\left[e_{i}, u\right]+\left[e_{j}, e_{k}\right] v\left[e_{i}, u\right]=} \\
e_{k}\left(\left[e_{i}, e_{j}\right][u, v]+\left[e_{j}, v\right]\left[e_{i}, u\right]\right)+v\left(\left[e_{i}, e_{j}\right]\left[u, e_{k}\right]+\left[e_{j}, e_{k}\right]\left[e_{i}, u\right]\right)
\end{gathered}
$$

since $e_{k}, v$ commute with the commutators (by the outer induction hypothesis). We are thus left with proving that $\left[e_{i}, e_{j}\right]\left[u, e_{k}\right]+\left[e_{j}, e_{k}\right]\left[e_{i}, u\right]=0$, which also serves as the basis of the (inner) induction. But this is exactly Lemma 3.7

We are now left with proving the other direction of Theorem 3.5.

Remark 3.9 ([BR05, Lemmas 3.43 and 3.44]). The identities

$$
\begin{aligned}
{[x, u][v, z]+[x, v][u, z] } & =0, \\
{[x, y][y, z] } & =0
\end{aligned}
$$

are consequences of the Grassmann identity.

Lemma 3.10. All identities of $\mathfrak{G}$ are consequences of the Grassmann identity.

Proof. We would first like to reduce to the multi-homogenous case. So, note that $\mathfrak{G} /\left\langle\varepsilon_{i} \mid i \in X\right\rangle$, for all finite $X \subseteq \mathbb{N}$, is isomorphic to $C^{\prime}\left[\lambda_{i} \mid i \in X\right] \otimes_{C} \mathfrak{G}$, where $C^{\prime}\left[\lambda_{i} \mid i \in X\right]$ is a commutative polynomial algebra in $|X|$ variables over $C^{\prime}=$ $C\left[\theta \mid \theta^{2}=2\right]$. Thus, if $f\left(x_{1}, \ldots, x_{n}\right)$ is an identity, then $f\left(\lambda_{1} \otimes x_{1}\right.$, $\left.\lambda_{2} \otimes x_{2}, \ldots, \lambda_{n} \otimes x_{n}\right)$ is also an identity. If we let $f_{d_{1}, \ldots, d_{n}}\left(\lambda_{1} \otimes x_{1}, \ldots, \lambda_{n} \otimes x_{n}\right)$ be the component of $f\left(\lambda_{1} \otimes x_{1}, \ldots, \lambda_{n} \otimes x_{n}\right)$ of degree $d_{i}$ in $\lambda_{i}$, we see that $f_{d_{1}, \ldots, d_{n}}\left(\lambda_{1} \otimes x_{1}, \ldots, \lambda_{n} \otimes x_{n}\right)=\lambda_{1}^{d_{1}} \cdots \lambda_{n}^{d_{n}} \otimes f_{d_{1}, \ldots, d_{n}}\left(x_{1}, \ldots, x_{n}\right)$ are the multihomogenous components of $f$, and must be equal to zero separately. Thus, we can assume that $f$ is multi-homogenous. 
So, let $f$ be a multi-homogenous identity of $\mathfrak{G}$. We need to prove that it is a consequence of the Grassmann identity. Since commutators are central, $f$ can be rewritten as a sum of terms of the form

$$
a x_{k_{1}} \cdots x_{k_{m}}\left[x_{k_{m+1}}, x_{k_{m+2}}\right]\left[x_{k_{m+3}}, x_{k_{m+4}}\right] \cdots\left[x_{k_{n-1}}, x_{k_{n}}\right],
$$

where $k_{1} \leq \cdots \leq k_{m}$. Using (15), we may assume that $k_{m+1}<\cdots<k_{n}$.

Substitution of 1 for all of $x_{1}, \ldots, x_{n}$ sends $f$ to the coefficient of the term $x_{1} \cdots x_{n}$, and since $f$ is an identity, this coefficient is zero. For every pair of variables $x_{i}, x_{j}$, substitute 1 for the other variables and $e_{1}, e_{2}$ for $x_{i}, x_{j}$; the only nonzero term is the one in which exactly these two variables are in the commutator, which again proves that the coefficient of this term is zero. Repeating this argument for all subsets of four variables, then six, and so on, we see that $f$ is zero modulo the Grassmann identity.

3.2. The ring $C[\varepsilon]$ and the connection to the Grassmann algebra. Our next goal is to show that when 2 is invertible, $C[\varepsilon]$ has enough idempotents to break $\mathfrak{G}$ into a sum of supercommutative pieces. The basic observation is that the expressions $\frac{1}{2} \theta \varepsilon_{i}$ (if defined) are idempotents.

Definition 3.11. For any subset $X \subseteq \mathbb{N}$, let $\mathfrak{G}_{X}=C\left\langle e_{j}, \varepsilon_{j}, \theta \mid j \in X\right\rangle \subset \mathfrak{G}$ be the subalgebra generated by all generators $\varepsilon_{j}$ and $e_{j}$ whose indices are in $X$.

Definition 3.12. Assume that 2 is invertible in $C$, and let $X \subseteq \mathbb{N}$ be a finite subset. For any association $s: X \rightarrow\{ \pm 1\}$ of signs to the indices in $X$, define

$$
\Lambda_{s}=\prod_{s(a)=-1} \frac{1}{2} \theta \varepsilon_{a} \prod_{s(b)=+1}\left(1-\frac{1}{2} \theta \varepsilon_{b}\right) .
$$

Proposition 3.13. Assume that 2 is invertible in $C$. Let $X \subseteq \mathbb{N}$ be a finite subset.

(1) The elements $\Lambda_{s} \in C[\varepsilon]$, for $s: X \rightarrow\{ \pm 1\}$, form a system of idempotents of $C[\varepsilon]$ whose sum is 1 .

(2) For every $s: X \rightarrow\{ \pm 1\}$, the algebra $\Lambda_{s} \mathfrak{G}_{X}$ is a free supercommutative algebra, with even generators $\theta$ and $\Lambda_{s} e_{b}$ for $s(b)=+1$, and odd generators $\Lambda_{s} e_{a}$ for $s(a)=-1$.

Proof. The defining relations imply that the elements $\frac{1}{2} \theta \varepsilon_{i}$ are idempotents, from which it follows that every $\Lambda_{s}$ is an idempotent. Furthermore

$$
\sum_{s: X \rightarrow\{ \pm 1\}} \Lambda_{s}=\prod_{i \in X}\left(\left(\frac{1}{2} \varepsilon_{i} \theta\right)+\left(1-\frac{1}{2} \varepsilon_{i} \theta\right)\right)=1 .
$$

For (2), let $a, a^{\prime}, b, b^{\prime} \in X$ be such that $s(a)=s\left(a^{\prime}\right)=-1$ and $s(b)=s\left(b^{\prime}\right)=+1$. We have that

$$
\begin{aligned}
{\left[e_{a} \Lambda_{s}, e_{a^{\prime}} \Lambda_{s}\right] } & =\varepsilon_{a} \varepsilon_{a^{\prime}} e_{a} e_{a^{\prime}} \Lambda_{s} \\
& =\varepsilon_{a} \varepsilon_{a^{\prime}} e_{a} e_{a^{\prime}} \frac{1}{2} \theta \varepsilon_{a} \frac{1}{2} \theta \varepsilon_{a^{\prime}} \Lambda_{s} \\
& =\frac{1}{4} \theta^{2} \varepsilon_{a}^{2} \varepsilon_{a^{\prime}}^{2} e_{a} e_{a^{\prime}} \Lambda_{s} \\
& =\theta^{2} \frac{1}{2} \theta \varepsilon_{a} \frac{1}{2} \theta \varepsilon_{a^{\prime}} e_{a} e_{a^{\prime}} \Lambda_{s} \\
& =2 e_{a} e_{a^{\prime}} \Lambda_{s},
\end{aligned}
$$


so $e_{a} \Lambda_{s}$ and $e_{a^{\prime}} \Lambda_{s}$ anticommute. The proof that $\Lambda_{s} e_{b}$ are central is analogous. Freeness then easily follows.

Multiplying by a suitable idempotent, we may thus declare finitely many of the $e_{1}, e_{2}, \ldots$ even, and finitely many others, odd. With this new understanding, we can prove a much stronger correspondence between $\mathfrak{G}$ and $S$ :

Theorem 3.14. Assume that 2 is invertible in $C$. For any $C$-algebra $A$ we have that $\mathrm{id}\left(A \otimes_{C} S\right)=\mathrm{id}\left(A \otimes_{C} \mathfrak{G}\right)$. In particular, id $\left(\mathrm{M}_{n}(S)\right)=\mathrm{id}\left(\mathrm{M}_{n}(\mathfrak{G})\right)$.

Proof. We first show that any identity of $A \otimes \mathfrak{G}$ is an identity of $A \otimes S$. Indeed, define a homomorphism of $C$-algebras, $\phi: S \rightarrow \mathfrak{G}$, by $\phi\left(e_{a}\right)=\frac{1}{2} \theta \varepsilon_{a} e_{a} \in \mathfrak{G}$ for odd generators $e_{a}$, and $\phi\left(e_{b}\right)=\left(1-\frac{1}{2} \theta \varepsilon_{b}\right) e_{b} \in \mathfrak{G}$ for even generators $e_{b}$ (note that the $e_{i}$ on the left hand side of this equation are elements from $S$, and on the right hand side from $\mathfrak{G}$ ). This homomorphism is clearly injective. Since $S, \mathfrak{G}$ and the image of $\phi$ are all free $C$-modules, and the image of a base of $S$ under $\phi$ can be completed to a base of $\mathfrak{G}$ (by considering the base of words in $S$, and the base of words multiplied by all idempotents associated to generators in the word, possibly times $\theta$ ), we see that the map $1_{A} \otimes \phi: A \otimes S \rightarrow A \otimes \mathfrak{G}$ is an injective homomorphism (indeed $\mathfrak{G} / \phi(S)$ is a free $C$-module, so $\left.\operatorname{Tor}_{1}^{C}(A, \mathfrak{G} / \phi(S))=0\right)$. Thus, $\operatorname{id}\left(A \otimes_{C} S\right) \supseteq \operatorname{id}\left(A \otimes_{C} \mathfrak{G}\right)$.

In the other direction, let $f \in \operatorname{id}\left(A \otimes_{C} S\right)$, and let $x_{i} \mapsto \hat{x}_{i} \in \mathfrak{G}$ be a substitution of elements from $\mathfrak{G}$ in the variables appearing in $f$. Let $X$ be the (finite) collection of all the indices $j$ of all $e_{j}$ or $\varepsilon_{j}$ appearing in some of the $\hat{x}_{i}$. Recall the definition of the subalgebra $\mathfrak{G}_{X}=C\left\langle e_{j}, \varepsilon_{j}, \theta \mid j \in X\right\rangle \subset \mathfrak{G}$. By Proposition 3.13, the idempotents $\Lambda_{s}$, with $s: X \rightarrow\{ \pm 1\}$, form a complete set of idempotents for $\mathfrak{G}$ (and thus $\mathfrak{G}_{X}$ ). Then it is sufficient to consider substitutions $x_{i} \mapsto \Lambda_{s} \hat{x}_{i} \in \Lambda_{s} \mathfrak{G}_{X}$ for some fixed $s: X \rightarrow\{ \pm 1\}$. But now, Proposition 3.13 shows that $\Lambda_{s} \mathfrak{G}_{X}$ is a free supercommutative algebra, so we can fix a canonical embedding $\psi: \Lambda_{s} \mathfrak{G}_{X} \rightarrow S$ of $\Lambda_{s} \mathfrak{G}_{X}$ in $S$. Again, we see that it maps the base of $\Lambda_{s} \mathfrak{G}_{X}$ into a set that can be completed to a base of $S$ (take the base generated by $\Lambda_{s}$ times words in $\mathfrak{G}_{X}$, and the base of words in $S$ ). Hence, the map

$$
\operatorname{id}_{A} \otimes \psi: A \otimes \Lambda_{s} \mathfrak{G}_{X} \rightarrow A \otimes S
$$

is an injective homomorphism. Thus, $f$ is zero on substitutions from $\Lambda_{s} \mathfrak{G}_{X}$ and is therefore zero on the substitution $x_{i} \mapsto \Lambda_{s} \hat{x}_{i} \in \Lambda_{s} \mathfrak{G}_{X}$.

Corollary 3.15. Suppose that 2 is invertible in $C$. Then the ideal of identities of the free supercommutative algebra, id $(S)$, is generated as a T-ideal by the Grassmann identity.

Remark 3.16. Over a field $C=\mathbb{F}$, the fact that $\operatorname{id}\left(A \otimes_{C} S\right)=\operatorname{id}\left(A \otimes_{C} \mathfrak{G}\right)$ would follow from the case $A=\mathbb{F}$, i.e. $\operatorname{id}(S)=\operatorname{id}(\mathfrak{G})$, since all $\mathbb{F}$-modules are flat.

Remark 3.17. Over a finite field, id $(G)$ strictly contains id $(S)$. For example over $C=\mathbb{F}_{3}$, the polynomial $x^{9} y^{3}-x^{3} y^{9}$ is an identity of $G$, which does not follow from the Grassmann identity.

Indeed, working modulo 3 , if $x=x_{0}+x_{1}$ is the decomposition of $x$ to homogenous parts, then $x^{3}=x_{0}^{3}+x_{1}^{3}=x_{0}^{3}$. But, the even part of $G$ is spanned by 1 and words of positive even length, so writing $x_{0}=\lambda+w$, where $\lambda \in \mathbb{F}_{3}$, we have that $x^{3}=x_{0}^{3}=\lambda^{3}=\lambda$. Thus, the identity becomes $\lambda \mu\left(\lambda^{2}-\mu^{2}\right)$, which is an identity of $\mathbb{F}_{3}$. A similar construction works over any finite field. 
As an immediate corollary, we now have a proof of the following theorem, proved by Regev and Krakowsky in characteristic 0 KR73, and by Giambruno and Koshlukov in characteristic $p \neq 2$ GK01.

Corollary 3.18. Suppose that 2 is invertible in $C$. Then the ideal of identities of the free supercommutative algebra, $\mathrm{id}(S)$, is generated as a T-ideal by the Grassmann identity.

Proof. According to Theorem 3.14, in this case id $(S)=\mathrm{id}(\mathfrak{G})$. But we have already seen that $\operatorname{id}(\mathfrak{G})$ is generated by the Grassmann identity (see Theorem 3.5).

3.3. Generalized signs. Now that we have a clear understanding of the role taken by the $\varepsilon_{i}$ 's, we can introduce some helpful notation. If $w \in \mathfrak{G}$ is a word in the generators, $w=e_{i_{1}} \cdots e_{i_{n}}$, then define: $\varepsilon_{w}=\varepsilon_{i_{1}}+\cdots+\varepsilon_{i_{n}}$. Clearly, for any two such words $w$ and $w^{\prime}$, we have $\varepsilon_{w} \varepsilon_{w^{\prime}} \in \operatorname{span}_{\mathbb{Z} / 2 \mathbb{Z}}\left\{\varepsilon_{i} \varepsilon_{j}\right\}$.

Definition 3.19. Define the map

$$
\exp : \operatorname{span}_{\mathbb{Z} / 2 \mathbb{Z}}\left\{\varepsilon_{i} \varepsilon_{j} \mid i, j \in \mathbb{N}\right\} \rightarrow C[\varepsilon]^{\times}
$$

by

$$
\exp \left(\sum \alpha_{i j} \varepsilon_{i} \varepsilon_{j}\right)=\prod_{i j}\left(1-\varepsilon_{i} \varepsilon_{j}\right)^{\alpha_{i j}}
$$

Remark 3.20. The exponent, a-priori defined on $\operatorname{span}_{\mathbb{Z}}\left\{\varepsilon_{i} \varepsilon_{j} \mid i, j \in \mathbb{N}\right\}$, is well defined over $\mathbb{Z} / 2 \mathbb{Z}$ because $\exp \left(2 \varepsilon_{i} \varepsilon_{j}\right)=\left(1-\varepsilon_{i} \varepsilon_{j}\right)^{2}=1-2 \varepsilon_{i} \varepsilon_{j}+\varepsilon_{i}^{2} \varepsilon_{j}^{2}=1-2 \varepsilon_{i} \varepsilon_{j}+$ $2 \varepsilon_{i} \varepsilon_{j}=1$. Thus

$$
\exp (a+b)=\exp (a) \exp (b)
$$

for any $a, b \in \operatorname{span}_{\mathbb{Z} / 2 \mathbb{Z}}\left\{\varepsilon_{i} \varepsilon_{j}\right\}$. For the same reason, $\exp (a)^{2}=\exp (2 a)=1$ for every $a$.

The following computation generalizes Equation (4).

Proposition 3.21. For any two monomials $u, w \in \mathfrak{G}$ in the generators $e_{i}$,

$$
u w=\exp \left(\varepsilon_{u} \varepsilon_{w}\right) w u .
$$

Proof. Equation (44) proves the case $u=e_{i}, w=e_{j}$. Let us verify the claim for $u=e_{i}, w=e_{j_{1}} \cdots e_{l_{m}}$. Indeed, we see that

$$
\begin{aligned}
u w=e_{i} e_{j_{1}} e_{j_{2}} \cdots e_{j_{m}} & =\exp \left(\varepsilon_{i} \varepsilon_{j_{1}}\right) e_{j_{1}} e_{i} e_{j_{2}} \cdots e_{j_{m}} \\
& =\exp \left(\varepsilon_{i} \varepsilon_{j_{1}}\right) \exp \left(\varepsilon_{i} \varepsilon_{j_{2}}\right) e_{j_{1}} e_{j_{2}} e_{i} \cdots e_{j_{m}}=\ldots \\
& =\exp \left(\varepsilon_{i} \varepsilon_{j_{1}}\right) \exp \left(\varepsilon_{i} \varepsilon_{j_{2}}\right) \cdots \exp \left(\varepsilon_{i} \varepsilon_{j_{m}}\right) e_{j_{1}} e_{j_{2}} \cdots e_{j_{m}} e_{i} \\
& =\exp \left(\varepsilon_{i}\left(\varepsilon_{j_{1}}+\cdots+\varepsilon_{j_{m}}\right)\right) w u \\
& =\exp \left(\varepsilon_{u} \varepsilon_{w}\right) w u
\end{aligned}
$$

Now, let $u=e_{i_{1}} \cdots e_{i_{n}}, w=e_{j_{1}} \cdots e_{j_{m}}$. Then:

$$
\begin{aligned}
u w & =e_{i_{1}} \cdots e_{i_{n-1}} e_{i_{n}} w \\
& =\exp \left(\varepsilon_{i_{n}} \varepsilon_{w}\right) e_{i_{1}} \cdots e_{i_{n-1}} w e_{i_{n}}=\exp \left(\varepsilon_{i_{n}} \varepsilon_{w}\right) \exp \left(\varepsilon_{i_{n-1}} \varepsilon_{w}\right) e_{i_{1}} \cdots w e_{i_{n-1}} e_{i_{n}} \\
& =\cdots=\exp \left(\varepsilon_{i_{n}} \varepsilon_{w}\right) \cdots \exp \left(\varepsilon_{i_{1}} \varepsilon_{w}\right) w e_{i_{1}} \cdots e_{i_{n}}=\exp \left(\varepsilon_{u} \varepsilon_{w}\right) w u
\end{aligned}
$$


Let us introduce a further generalization of the exponent map, which we call a generalized sign. We use the natural action of the infinite symmetric group $S_{\mathbb{N}}$ on $C[\varepsilon]$ by $\phi_{\sigma}(\theta)=\theta$ and

$$
\phi_{\sigma}\left(\varepsilon_{i}\right)=\varepsilon_{\sigma(i)} .
$$

Definition 3.22. Let $w=\left(w_{1}, \ldots, w_{n}\right)$ be an $n$-tuple of words in the generators $e_{i}$. For $\sigma \in S_{n}$, a permutation on the set $\{1, \ldots, n\}$, we define the generalized sign to be:

$$
\mathfrak{s} \mathfrak{g n} \mathfrak{n}_{w}(\sigma)=\exp \left(\sum_{\substack{i<j \\ \sigma(i)>\sigma(j)}} \varepsilon_{w_{\sigma(i)}} \varepsilon_{w_{\sigma(j)}}\right)
$$

Proposition 3.23. Let $w=\left(w_{1}, \ldots, w_{n}\right)$ be an $n$-tuple of words in the generators $e_{i}$.

(1) For every $\sigma \in S_{n}$,

$$
w_{\sigma(1)} w_{\sigma(2)} \cdots w_{\sigma(n)}=\mathfrak{s g n}_{w}(\sigma) w_{1} w_{2} \cdots w_{n} .
$$

(2) For every $\sigma, \tau \in S_{n}$,

$$
\mathfrak{s g \mathfrak { n }}{ }_{w}(\sigma \tau)=\mathfrak{s g \mathfrak { n }}{ }_{w}(\sigma) \mathfrak{s g \mathfrak { n }}{ }_{\sigma(w)}(\tau)
$$

where $\sigma(w)=\left(w_{\sigma(1)}, \ldots, w_{\sigma(n)}\right)$.

(3) In particular, when $w=\left(e_{1}, \ldots, e_{n}\right)$,

$$
\mathfrak{s g n}_{w}(\sigma \tau)=\mathfrak{s g n}_{w}(\sigma) \phi_{\sigma}\left(\mathfrak{s g n} \mathfrak{n}_{w}(\tau)\right)
$$

Proof. Write $\sigma=s_{1} \cdots s_{m}$ where $s_{j}=\left(k_{j}, k_{j}+1\right)$ are Coxeter generators of $S_{n}$. We prove (1) by induction on $m$. For $m=0$, the claim is trivial. Assume the claim holds for $\pi=s_{1} \cdots s_{m-1}$. Then according to Proposition 3.21 and since $s_{m}$ transposes $w_{\pi\left(k_{m}\right)}$ and $w_{\pi\left(k_{m}+1\right)}$, we have:

$$
\begin{aligned}
w_{\sigma(1)} & w_{\sigma(2)} \cdots w_{\sigma(n)}=w_{\pi s_{m}(1)} w_{\pi s_{m}(2)} \cdots w_{\pi s_{m}(n)} \\
& =w_{\pi(1)} w_{\pi(2)} \cdots w_{\pi\left(k_{m}-1\right)} w_{\pi\left(k_{m}+1\right)} w_{\pi\left(k_{m}\right)} w_{\pi\left(k_{m}+2\right)} \cdots w_{\pi(n)} \\
& =\exp \left(\varepsilon_{w_{\pi\left(k_{m}\right)}} \varepsilon_{w_{\pi\left(k_{m}+1\right)}}\right) w_{\pi(1)} w_{\pi(2)} \cdots w_{\pi\left(k_{m}-1\right)} w_{\pi\left(k_{m}\right)} w_{\pi\left(k_{m}+1\right)} \cdots w_{\pi(n)} \\
& =\exp \left(\varepsilon_{w_{\sigma\left(k_{m}\right)}} \varepsilon_{w_{\sigma\left(k_{m}+1\right)}}\right) w_{\pi(1)} w_{\pi(2)} \cdots w_{\pi(n)} \\
& =\exp \left(\varepsilon_{w_{\sigma\left(k_{m}\right)}} \varepsilon_{w_{\sigma\left(k_{m}+1\right)}}\right) \mathfrak{s g \mathfrak { g n } _ { w }}(\pi) w_{1} w_{2} \cdots w_{n},
\end{aligned}
$$

where the last equality follows from the induction hypothesis. Acting by $s_{m}=$ $\left(k_{m}, k_{m}+1\right)$ does not affect the order of any of the pairs $i<j$, except for flipping 
the order of the pair $k_{m}, k_{m}+1$. Thus,

$$
\begin{aligned}
& \exp \left(\varepsilon_{w_{\sigma\left(k_{m}\right)}} \varepsilon_{w_{\sigma\left(k_{m}+1\right)}}\right) \mathfrak{s g \mathfrak { n }} \mathfrak{w}_{w}(\pi) \\
& =\exp \left(\varepsilon_{w_{\sigma\left(k_{m}\right)}} \varepsilon_{w_{\sigma\left(k_{m}+1\right)}}\right) \exp \left(\sum_{\substack{i<j \\
\pi(i)>\pi(j)}} \varepsilon_{w_{\pi(i)}} \varepsilon_{w_{\pi(j)}}\right) \\
& =\exp \left(\varepsilon_{w_{\sigma\left(k_{m}\right)}} \varepsilon_{w_{\sigma\left(k_{m}+1\right)}}+\sum_{\substack{s_{m}(i)<s_{m}(j) \\
\pi s_{m}(i)>s_{m}(j)}} \varepsilon_{w_{\pi s_{m}(i)}} \varepsilon_{w_{\pi s_{m}(j)}}\right) \\
& =\exp \left(\varepsilon_{w_{\sigma\left(k_{m}\right)}} \varepsilon_{w_{\sigma\left(k_{m}+1\right)}}+\sum_{\substack{s_{m}(i)<s_{m}(j) \\
\sigma(i)>\sigma(j)}} \varepsilon_{w_{\sigma(i)}} \varepsilon_{w_{\sigma(j)}}\right) \\
& =\exp \left(\sum_{\substack{i<j \\
\sigma(i)>\sigma(j)}} \varepsilon_{w_{\sigma(i)}} \varepsilon_{w_{\sigma(j)}}\right)=\mathfrak{s g n}_{w}(\sigma),
\end{aligned}
$$

as claimed.

To prove (2), we compute

$$
\begin{aligned}
\mathfrak{s g n}_{w}(\sigma) \mathfrak{s} \mathfrak{g n}_{\sigma(w)}(\tau) & =\exp \left(\sum_{\substack{i<j \\
\sigma(i)>\sigma(j)}} \varepsilon_{w_{\sigma(i)}} \varepsilon_{w_{\sigma(j)}}+\sum_{\substack{i<j \\
\tau(i)>\tau(j)}} \varepsilon_{\sigma(w)_{\tau(i)}} \varepsilon_{\sigma(w)_{\tau(j)}}\right) \\
& =\exp \left(\sum_{\substack{i<j \\
\sigma(i)>\sigma(j)}} \varepsilon_{w_{\sigma(i)}} \varepsilon_{w_{\sigma(j)}}+\sum_{\substack{i<j \\
\tau(i)>\tau(j)}} \varepsilon_{w_{\sigma \tau(i)}} \varepsilon_{\left.w_{\sigma \tau(j)}\right)}\right) \\
& =\exp \left(\sum_{\substack{\tau(i)<\tau(j) \\
\sigma \tau(i)>\sigma \tau(j)}} \varepsilon_{w_{\sigma \tau(i)}} \varepsilon_{w_{\sigma \tau(j)}}+\sum_{\substack{i<j \\
\tau(i)>\tau(j)}} \varepsilon_{w_{\sigma \tau(i)}} \varepsilon_{w_{\sigma \tau(j)}}\right) .
\end{aligned}
$$

But since each pair $i<j$ whose order is inverted by $\sigma \tau$ is inverted by $\sigma$ or by $\tau$, and not both, we have that the latter sum is equal to

$$
\exp \left(\sum_{\substack{i<j \\ \sigma \tau(i)>\sigma \tau(j)}} \varepsilon_{w_{\sigma \tau(i)}} \varepsilon_{w_{\sigma \tau(j)}}\right)=\mathfrak{s g n}_{w}(\sigma \tau) .
$$

Since in the Grassmann algebra $G$ we have that $e_{\sigma(1)} \cdots e_{\sigma(n)}=\operatorname{sgn}(\sigma) e_{1} \cdots e_{n}$, Proposition 3.23. (1) shows that the generalized sign $\mathfrak{s g n}_{w}(\cdot)$ plays in $\mathfrak{G}$ the same role that the usual sign plays in $G$. Furthermore, the idempotent corresponding to 
the constant function $s(i)=-1(i=1, \ldots, n)$ satisfies

$$
\mathfrak{s g n}_{\left(e_{1}, \ldots, e_{n}\right)}(\sigma) \Lambda_{s}=\operatorname{sgn}(\sigma) \Lambda_{s}
$$

since the $e_{i}$ anticommute in the presence of $\Lambda_{s}$.

Remark 3.24. The generalized sign can be given a cohomological interpretation. Let $W$ denote the set of $n$-tuples of words, on which $S_{n}$ acts by $\sigma: w \mapsto \sigma^{-1}(w)$. Let $M$ be the multiplicative group of functions $W \rightarrow C[\varepsilon]^{\times}$, on which $S_{n}$ acts by $s \mapsto s \circ \sigma^{-1}$. Proposition 3.23. (10) is the claim that $\mathfrak{s g n}(\cdot): S_{n} \rightarrow M$, defined by $\mathfrak{s g n}(\sigma): w \mapsto \mathfrak{s g n}_{w}(\sigma)$, defines a cohomology class in $H^{1}\left(S_{n}, M\right)$.

3.4. The co-module sequence of $\mathfrak{G}$. We now turn our attention to the comodules and co-dimensions of $\mathfrak{G}$. We begin by defining an $S_{n}$-representation analogous to the usual sign representation.

Definition 3.25. Fix $w=\left(e_{1}, \ldots, e_{n}\right)$. We consider the natural action of $S_{n}$ on $C[\varepsilon]$ twisted by signs: For each $\sigma \in S_{n}$ and $\lambda \in C[\varepsilon]$,

$$
\sigma(\lambda)=\mathfrak{s g n}_{w}(\sigma) \phi_{\sigma}(\lambda) \text {. }
$$

Also let $C[\varepsilon]_{n}$ denote the $S_{n}$-submodule of $C[\varepsilon]$ generated as a module by $1 \in C[\varepsilon]$.

Remark 3.26. According to Proposition [3.23, (3), this indeed gives $C[\varepsilon]$ an $S_{n^{-}}$ module structure, as

$$
\begin{aligned}
(\sigma \tau)(\lambda) & =\mathfrak{s g n}_{w}(\sigma \tau) \phi_{\sigma \tau}(\lambda) \\
& =\mathfrak{s g n}_{w}(\sigma) \phi_{\sigma}\left(\mathfrak{s g n} \mathfrak{n}_{w}(\tau)\right) \phi_{\sigma}\left(\phi_{\tau}(\lambda)\right) \\
& =\mathfrak{s g n}_{w}(\sigma) \phi_{\sigma}\left(\mathfrak{s g n}_{w}(\tau) \phi_{\tau}(\lambda)\right) \\
& =\sigma\left(\mathfrak{s g \mathfrak { g }}{ }_{w}(\tau) \phi_{\tau}(\lambda)\right)=\sigma(\tau(\lambda)) .
\end{aligned}
$$

Example 3.27. Consider the $S_{3}$-module $C[\varepsilon]_{3}$. By definition $C[\varepsilon]_{3}$ is spanned as a $C$-module by the elements $\sigma(1)=\mathfrak{s g n}_{w}(\sigma)$, which are:

$$
\begin{aligned}
\mathfrak{s g n}_{w}(1) & =1 \\
\mathfrak{s g n}_{w}((12)) & =\exp \left(\varepsilon_{1} \varepsilon_{2}\right)=1-\varepsilon_{1} \varepsilon_{2}, \\
\mathfrak{s g n}_{w}((23)) & =\exp \left(\varepsilon_{2} \varepsilon_{3}\right)=1-\varepsilon_{2} \varepsilon_{3}, \\
\mathfrak{s g n}_{w}((13)) & =\exp \left(\varepsilon_{1} \varepsilon_{2}+\varepsilon_{2} \varepsilon_{3}+\varepsilon_{1} \varepsilon_{3}\right)=\left(1-\varepsilon_{1} \varepsilon_{2}\right)\left(1-\varepsilon_{2} \varepsilon_{3}\right)\left(1-\varepsilon_{1} \varepsilon_{3}\right) \\
& =1-\varepsilon_{1} \varepsilon_{2}-\varepsilon_{2} \varepsilon_{3}-\varepsilon_{1} \varepsilon_{3}+\theta \varepsilon_{1} \varepsilon_{2} \varepsilon_{3}, \\
\mathfrak{s g n}_{w}((123)) & =\exp \left(\varepsilon_{1}\left(\varepsilon_{2}+\varepsilon_{3}\right)\right)=\left(1-\varepsilon_{1} \varepsilon_{2}\right)\left(1-\varepsilon_{1} \varepsilon_{3}\right) \\
& =1-\varepsilon_{1} \varepsilon_{2}-\varepsilon_{1} \varepsilon_{3}+\theta \varepsilon_{1} \varepsilon_{2} \varepsilon_{3}, \\
\mathfrak{s g n}_{w}((132)) & =\exp \left(\varepsilon_{3}\left(\varepsilon_{1}+\varepsilon_{2}\right)\right)=\left(1-\varepsilon_{1} \varepsilon_{3}\right)\left(1-\varepsilon_{2} \varepsilon_{3}\right) \\
& =1-\varepsilon_{1} \varepsilon_{3}-\varepsilon_{2} \varepsilon_{3}+\theta \varepsilon_{1} \varepsilon_{2} \varepsilon_{3} .
\end{aligned}
$$

Therefore, $C[\varepsilon]_{3}$ is a free $C$-module of rank 4 , spanned by $1, \varepsilon_{1} \varepsilon_{2}, \varepsilon_{2} \varepsilon_{3}$ and $\varepsilon_{1} \varepsilon_{3}-$ $\theta \varepsilon_{1} \varepsilon_{2} \varepsilon_{3}$.

We can now state the main result of this section.

Theorem 3.28. The $n$-th co-module of $\mathfrak{G}$ is isomorphic, as an $S_{n}$-module, to $C[\varepsilon]_{n}$.

To prove the theorem, we will first establish that a multilinear polynomial that vanishes on $e_{1}, \ldots, e_{n}$ vanishes on any other substitution. Since $S_{n}$ acts on the space $P_{n}$ defined in (2) by reordering variables, and since reordering variables multiplies by the generalized sign, Theorem 3.28 follows (as will be explained below). 
We first observe that $\mathfrak{G}$ has plenty of endomorphisms.

Lemma 3.29. For any $n$-tuple of words $w=\left(w_{1}, \ldots, w_{n}\right)$ in the generators $e_{i}$, there is a morphism $\eta_{w}: \mathfrak{G} \rightarrow \mathfrak{G}$ such that for all $1 \leq i \leq n$ :

$$
\eta_{w}\left(e_{i}\right)=w_{i} \text {. }
$$

Proof. First we show that for every $\ell$ and for every word $w$ of length 1 or 2 , there is a homomorphism $\mathfrak{G} \rightarrow \mathfrak{G}$ of $C$-algebras such that $e_{i} \mapsto e_{i}(i \neq \ell)$ and $e_{\ell} \mapsto w$.

Indeed, when $w=e_{j}$, define the map on $C[\varepsilon]$ by $\theta \mapsto \theta, \varepsilon_{i} \mapsto \varepsilon_{i}$ for every $i \neq \ell$, and $\varepsilon_{l} \mapsto \varepsilon_{j}$. This is easily seen to be well defined.

Likewise when $w=e_{j} e_{k}$, define the map $\eta_{j, k, \ell}$ by $\theta \mapsto \theta, \varepsilon_{i} \mapsto \varepsilon_{i}, e_{i} \mapsto e_{i}$ for $i \neq \ell$, and $\varepsilon_{\ell} \mapsto \varepsilon_{j}+\varepsilon_{k}-\theta \varepsilon_{j} \varepsilon_{k}, e_{\ell} \mapsto e_{j} e_{k}$. In order to show that this homomorphism is well defined, it suffices to check that $\eta_{j, k, \ell}$ respects the relations $\varepsilon_{\ell}^{2}=\theta \varepsilon_{l}$ and $\left[e_{\ell}, e_{i}\right]=\varepsilon_{\ell} \varepsilon_{i} e_{\ell} e_{i}$ for all $i$. For the first relation we have

$$
\begin{aligned}
\eta_{j, k, \ell}\left(\varepsilon_{\ell}\right)^{2} & =\left(\varepsilon_{j}+\varepsilon_{k}-\theta \varepsilon_{j} \varepsilon_{k}\right)^{2} \\
& =\theta \varepsilon_{j}+\theta \varepsilon_{k}-2 \varepsilon_{j} \varepsilon_{k}=\theta\left(\varepsilon_{j}+\varepsilon_{k}-\theta \varepsilon_{j} \varepsilon_{k}\right)=\eta_{j, k, \ell}(\theta) \eta_{j, k, \ell}\left(\varepsilon_{\ell}\right) .
\end{aligned}
$$

As for the second relation, for $i=\ell$ we have

$$
\begin{aligned}
\eta_{j, k, \ell}\left(\varepsilon_{\ell}\right) \eta_{j, k, \ell}\left(\varepsilon_{\ell}\right) \eta_{j, k, \ell}\left(e_{\ell}\right) \eta_{j, k, \ell}\left(e_{\ell}\right) & =\left(\varepsilon_{j}+\varepsilon_{k}-\theta \varepsilon_{j} \varepsilon_{k}\right)^{2} e_{j} e_{k} e_{j} e_{k} \\
& =\theta\left(\varepsilon_{j}+\varepsilon_{k}-\theta \varepsilon_{j} \varepsilon_{k}\right)\left(1-\varepsilon_{j} \varepsilon_{k}\right) e_{j} e_{j} e_{k} e_{k} \\
& =\theta\left(\varepsilon_{j}+\varepsilon_{k}-\theta \varepsilon_{j} \varepsilon_{k}\right) e_{j}^{2} e_{k}^{2}=0=\eta_{j, k, \ell}\left(\left[e_{l}, e_{l}\right]\right)
\end{aligned}
$$

since $\theta \varepsilon_{j} e_{j}^{2}=\theta \varepsilon_{k} e_{k}^{2}=0$. For $i \neq \ell$,

$$
\begin{aligned}
\eta_{j, k, \ell}\left(\varepsilon_{\ell}\right) \eta_{j, k, \ell}\left(\varepsilon_{i}\right) \eta_{j, k, \ell}\left(e_{\ell}\right) \eta_{j, k, \ell}\left(e_{i}\right) & =\left(\varepsilon_{j}+\varepsilon_{k}-\theta \varepsilon_{j} \varepsilon_{k}\right) \varepsilon_{i} e_{j} e_{k} e_{i} \\
& =\left(\varepsilon_{j} \varepsilon_{i}+\varepsilon_{k} \varepsilon_{i}-\theta \varepsilon_{j} \varepsilon_{k} \varepsilon_{i}\right) e_{j} e_{k} e_{i} \\
& =\left(1-\left(1-\varepsilon_{j} \varepsilon_{i}\right)\left(1-\varepsilon_{k} \varepsilon_{i}\right)\right) e_{j} e_{k} e_{i} \\
& =\left(1-\exp \left(\varepsilon_{v} \varepsilon_{i}\right)\right) v e_{i}
\end{aligned}
$$

where $v=e_{j} e_{k}$. But by Proposition 3.21, we know that $\left(1-\exp \left(\varepsilon_{v} \varepsilon_{i}\right)\right) v e_{i}=$ $v e_{i}-e_{i} v=\left[v, e_{i}\right]=\left[\eta_{j, k, \ell}\left(e_{\ell}\right), \eta_{j, k, \ell}\left(e_{i}\right)\right]=\eta_{j, k, \ell}\left(\left[e_{\ell}, e_{i}\right]\right)$, so $\eta_{j, k, \ell}\left(\varepsilon_{\ell} \varepsilon_{i} e_{\ell} e_{i}\right)=$ $\eta_{j, k, \ell}\left(\left[e_{\ell}, e_{i}\right]\right)$, as we wanted to show.

Now compose the morphisms defined above so that each $e_{i}$ is mapped to a word of length len $\left(w_{i}\right)$ on distinct generators, and then map the generators to the respective letters in the $w_{i}$.

Lemma 3.30. Let $f\left(x_{1}, \ldots, x_{n}\right) \in P_{n}$ be any multilinear polynomial in noncommutative variables (with coefficients in $C$ ). Then $f$ is an identity of $\mathfrak{G}$ if and only if $f\left(e_{1}, \ldots, e_{n}\right)=0$.

Proof. If $f$ is an identity then obviously $f\left(e_{1}, \ldots, e_{n}\right)=0$. On the other hand assume $f\left(e_{1}, \ldots, e_{n}\right)=0$. For every $w_{1}, \ldots, w_{n}$ we have that

$$
f\left(w_{1}, \ldots, w_{n}\right)=\eta_{w}\left(f\left(e_{1}, \ldots, e_{n}\right)\right)=0,
$$

so we are done by multilinearity.

Proof of Theorem 3.28, Let $M_{n}=P_{n} /\left(\mathrm{id}(\mathfrak{G}) \cap P_{n}\right)$ denote the $n$-th co-module of $\mathfrak{G}$, where $P_{n}$ is defined in (2). Define a linear mapping $\mu: M_{n} \rightarrow C[\varepsilon]_{n} e_{1} \cdots e_{n}$ by the substitution $x_{i} \mapsto e_{i}$. By Proposition 3.23, $\sum_{\sigma \in S_{n}} a_{\sigma} x_{\sigma(1)} \cdots x_{\sigma(n)}$ is mapped to $\sum_{\sigma \in S_{n}} a_{\sigma} e_{\sigma(1)} \cdots e_{\sigma(n)}=\sum_{\sigma \in S_{n}} a_{\sigma} \mathfrak{s g n}_{w}(\sigma) e_{1} \cdots e_{n}$, where $w=\left(e_{1}, \ldots, e_{n}\right)$. 
Let $\nu: C[\varepsilon]_{n} e_{1} \cdots e_{n} \rightarrow C[\varepsilon]_{n}$ denote the isomorphism of $C$-modules defined by setting $\nu\left(\lambda e_{1} \cdots e_{n}\right)=\lambda$. Let $\psi=\nu \circ \mu: M_{n} \rightarrow C[\varepsilon]_{n}$. We will prove that $\psi$ is an isomorphism of $S_{n}$-modules.

Indeed, $\psi\left(\sum_{\sigma \in S_{n}} a_{\sigma} x_{\sigma(1)} \cdots x_{\sigma(n)}\right)=\sum_{\sigma \in S_{n}} a_{\sigma \mathfrak{s g n}}(\sigma)$. But, for every $\pi \in S_{n}$,

$$
\begin{aligned}
\psi \pi\left(\sum_{\sigma \in S_{n}} a_{\sigma} x_{\sigma(1)} \cdots x_{\sigma(n)}\right) & =\psi\left(\sum_{\sigma \in S_{n}} a_{\sigma} x_{\pi \sigma(1)} \cdots x_{\pi \sigma(n)}\right) \\
& =\sum_{\sigma \in S_{n}} a_{\sigma} \mathfrak{s g n}_{w}(\pi \sigma) \\
& =\mathfrak{s g \mathfrak { g }}{ }_{w}(\pi) \phi_{\pi}\left(\sum_{\sigma \in S_{n}} a_{\sigma} \mathfrak{s g n}_{w}(\sigma)\right) \\
& =\mathfrak{s g n}_{w}(\pi) \phi_{\pi}\left(\psi\left(\sum_{\sigma \in S_{n}} a_{\sigma} x_{\sigma(1)} \cdots x_{\sigma(n)}\right)\right) \\
& =\pi\left(\psi\left(\sum_{\sigma \in S_{n}} a_{\sigma} x_{\sigma(1)} \cdots x_{\sigma(n)}\right)\right),
\end{aligned}
$$

showing that $\psi$ is a homomorphism of $S_{n}$-modules.

Since $1=\psi\left(x_{1} \cdots x_{n}\right)$ generates $C[\varepsilon]_{n}, \psi$ is surjective. Injectivity follows once we show that if $f \in P_{n}$ becomes zero under the substitution $x_{i} \mapsto e_{i}$ then $f$ is an identity, which is the content of Lemma 3.30

In addition to having the co-modules of $\mathfrak{G}$, we can already calculate its codimensions:

Theorem 3.31. The $S_{n}$-module $C[\varepsilon]_{n}$ is a free $C$-module of rank $2^{n-1}$.

Proof. In the proof of Lemma 3.10 we have seen that modulo consequences of the Grassmann identity, every non-commutative polynomial $f$ of degree $n$, and in particular every multilinear polynomial $f$ of degree $n$, is a sum of elements of the form $x_{i_{1}} \cdots x_{i_{m}}\left[x_{i_{m+1}}, x_{i_{m+2}}\right] \cdots\left[x_{i_{n-1}}, x_{i_{n}}\right]$ where $i_{1} \leq \cdots \leq i_{m}$ and we can assume that $i_{m+1}<\cdots<i_{n}$. Therefore, those elements generate the $n$-th co-module of $\mathfrak{G}$ as a $C$-module. Thus, if we let

$$
\begin{aligned}
N=\operatorname{span}_{C}\left\{x_{i_{1}} \cdots x_{i_{m}}\left[x_{i_{m+1}}, x_{i_{m+2}}\right] \cdots\left[x_{i_{n-1}}, x_{i_{n}}\right] \mid\right. \\
\left.i_{1}<\cdots<i_{m}, i_{m+1}<\cdots<i_{n}\right\},
\end{aligned}
$$

then $N /(N \cap \operatorname{id}(\mathfrak{G}))$ is the $n$-th co-module of $\mathfrak{G}$, which is (by Theorem 3.28) isomorphic to $C[\varepsilon]_{n}$. Hence, $C[\varepsilon]_{n}$ is the quotient of $N$ by all identities of $\mathfrak{G}$. But, we have seen in the proof of Lemma 3.10 that all identities of $\mathfrak{G}$ in $N$ are zero, and hence $N$ is isomorphic to $C[\varepsilon]_{n}$.

However, there are exactly $2^{n-1}$ polynomials in the set spanning $N$, and we have already seen that they are linearly independent: indeed, in the proof of Lemma 3.10 . we have shown that if $\sum a_{i} x_{i_{1}} \cdots x_{i_{m}}\left[x_{i_{m+1}}, x_{i_{m+2}}\right] \cdots\left[x_{i_{n-1}}, x_{i_{n}}\right] \in N$ is an identity (in particular, a linear relation among the generators of $N$ ), then the coefficients $a_{i}$ are zero. Hence, they are linearly independent.

Corollary 3.32. For any field $C=\mathbb{F}$ of any characteristic, the co-dimension sequence of $\mathfrak{G}$ is $c_{n}(\mathfrak{G})=2^{n-1}$.

An immediate consequence is that we know the co-dimension of $G$, the usual Grassmann algebra, for any field of characteristic different than 2, generalizing 
the well known classical result in characteristic 0 (see also [LPT05] for a purely combinatoric proof).

Corollary 3.33. For any field $\mathbb{F}$ with char $\mathbb{F} \neq 2$, we have $c_{n}(G)=2^{n-1}$.

Proof. We have shown that when 2 is invertible, $\operatorname{id}(S)=\mathrm{id}(\mathfrak{G})$ (see Theorem 3.14), and since $S$ is an extension by scalars of $G$ they have the same co-dimension.

\section{Generalized superalgebras}

4.1. Generalized superalgebras. Now that we have the basic machinery of the generalized Grassmann algebra, we use it to replicate the success of the standard Grassmann algebra in characteristic 0. The first problem is that while the Grassmann algebra $G$ has a natural superalgebra structure, given by the words of even and odd length, the even-odd grading on $\mathfrak{G}$ is uninteresting, as exemplified by Lemma 3.29

Recall the definition of $C[\varepsilon]$ in Definition 3.1. Taking advantage of the many idempotents of $C[\varepsilon]$, we choose the following grading.

We denote the countable abelian group of exponent $2,(\mathbb{Z} / 2 \mathbb{Z})^{\oplus \mathbb{N}}$, by $2^{<\omega}$.

Definition 4.1. A $C[\varepsilon]$-algebra is called a $\Sigma$-superalgebra over $C$ if it is graded by $2^{<\omega}$.

Our first example is the algebra $\mathfrak{G}$ itself:

Definition 4.2. The extended Grassmann algebra is $2^{<\omega}$-graded by letting $C[\varepsilon]$ be contained in the zero component, and setting the grade of each $e_{i}$ to be $(0, \ldots, 0$, $1,0, \ldots)$ where the 1 is in the $i$-th component. The degree of a word $w \in \mathfrak{G}$ is $g=\left(\operatorname{deg}_{1} w, \operatorname{deg}_{2} w, \ldots\right)$ modulo 2 , where $\operatorname{deg}_{i} w$ is the number of occurrences of $e_{i}$ in $w$.

The zero component is thus $\mathfrak{G}_{0}=C[\varepsilon]\left[e_{1}^{2}, e_{2}^{2}, \ldots\right]$, which is contained in the center of $\mathfrak{G}$. For every $g=\left(g_{1}, g_{2}, \ldots\right) \in 2^{<\omega}$, which is eventually zero by definition, let $e_{g}=\prod e_{i}^{g_{i}}$ and $\varepsilon_{g}=g_{1} \varepsilon_{1}+g_{2} \varepsilon_{2}+\cdots \in \operatorname{span}_{\mathbb{Z} / 2 \mathbb{Z}}\left\{\varepsilon_{i}\right\}$. The corresponding component $\mathfrak{G}_{g}=\mathfrak{G}_{0} e_{g}$ is a rank 1 module over $\mathfrak{G}_{0}$, so the grading is "thin".

Definition 4.3. Let $\mathfrak{A}=\bigoplus_{g \in 2<\omega} \mathfrak{A}_{g}$ be any $\Sigma$-superalgebra over $C$. We define the $\Sigma$-supercommutator $\{a, b\} \in \mathfrak{A}$ for homogenous elements $a \in \mathfrak{A}_{g}$ and $b \in \mathfrak{A}_{h}$ by setting

$$
\{a, b\}=a b-\exp \left(\varepsilon_{g} \varepsilon_{h}\right) b a,
$$

extended bilinearly to all $a, b \in \mathfrak{A}$.

We say that $\mathfrak{A}$ is $\Sigma$-supercommutative if $\{a, b\}=0$ for all $a, b \in \mathfrak{A}$.

Example 4.4. The extended Grassmann algebra $\mathfrak{G}$ is $\Sigma$-supercommutative. Indeed, by Proposition 3.21 for any pair of words $u \in \mathfrak{G}_{g}$ and $v \in \mathfrak{G}_{h}$ we have that $u v=\exp \left(\varepsilon_{g} \varepsilon_{h}\right) v u=\exp \left(\varepsilon_{g} \varepsilon_{h}\right) v u$, or in other words, $\{u, v\}=0$.

We will use regular font for the standard supertheoretic notions, such as sgn (.), sCent, str, $A, B, C, G$, and the Fraktur font for the corresponding $\Sigma$-supertheory notions, $\mathfrak{s g n}(\cdot), \mathfrak{s C \mathfrak { C }} \mathfrak{n}, \mathfrak{s t r}, \mathfrak{A}, \mathfrak{B}, \mathfrak{C}, \mathfrak{G}$, etc. 
Example 4.5. As another example, one can consider $\mathfrak{S}$, the free $\Sigma$-supercommutative $\Sigma$-superalgebra on the generators $e_{g}^{(n)}(n=1,2, \ldots)$ where $e_{g}^{(n)} \in \mathfrak{S}_{g}$ is a homogenous generator of the component with degree $g$. As a result, $\mathfrak{S}$ is generated by the generators $e_{g}^{(n)}$ under the relations:

$$
\left[e_{g}^{(n)}, e_{h}^{(m)}\right]=\left(1-\exp \left(\varepsilon_{g} \varepsilon_{h}\right)\right) e_{g}^{(n)} e_{h}^{(m)} .
$$

Note that $\operatorname{id}(\mathfrak{G})=\operatorname{id}(\mathfrak{S})$, because $\mathfrak{G} \subset \mathfrak{S}$ and $\mathfrak{S}$ satisfies the Grassmann identity.

4.2. The generalized Grassmann hull. Now that we have an appropriate grading, we can generalize the Grassmann hull of an algebra (see Theorem 1.5 for the notion of the Grassmann hull for superalgebras). Similarly to the standard Grassmann hull, one can use either the Grassmann algebra or the free $\Sigma$-supercommutative algebra to define it (for an example in the case of char $=0$, see [GZ05, p. 83-85]). For our purposes, it will be more convenient to use the free $\Sigma$-supercommutative algebra.

Definition 4.6. Let $\mathfrak{A}=\bigoplus_{g \in 2<\omega} \mathfrak{A}_{g}$ be a $\Sigma$-superalgebra. The generalized Grassmann hull of $\mathfrak{A}$ is by definition

$$
\mathfrak{S}[\mathfrak{A}]=\bigoplus_{g \in 2<\omega}\left(\mathfrak{S}_{g} \otimes_{C[\varepsilon]} \mathfrak{A}_{g}\right)
$$

with the $2^{<\omega}$-grading defined by $\mathfrak{S}[\mathfrak{A}]_{g}=\mathfrak{S}_{g} \otimes_{C[\varepsilon]} \mathfrak{A}_{g}$.

Example 4.7. Let $A$ be any $C$-algebra. Tensoring with the $C[\varepsilon]$-group algebra $C[\varepsilon]\left[2^{<\omega}\right]$, which is naturally a $\Sigma$-superalgebra over $C$, gives $A \otimes_{C} C[\varepsilon]\left[2^{<\omega}\right]$ a natural $\Sigma$-superalgebra grading, where the homogeneous components are

$$
\left(A \otimes_{C} C[\varepsilon]\left[2^{<\omega}\right]\right)_{g}=A \otimes_{C}\left(C[\varepsilon]\left[2^{<\omega}\right]\right)_{g}
$$

and

$$
A \otimes_{C} \mathfrak{S}=\mathfrak{S}\left[A \otimes_{C} C[\varepsilon]\left[2^{<\omega}\right]\right] .
$$

We will now define the notion of a $\Sigma$-superidentity:

Definition 4.8. Define $C[\varepsilon]\left\langle x_{1}^{(g)}, x_{2}^{(g)}, \ldots \mid g \in 2^{<\omega}\right\rangle$, denoted by $C[\varepsilon]\left\langle X^{(g)}\right\rangle$ for brevity, to be the free $\Sigma$-superalgebra on countably many generators in each degree. The elements of this algebra are called $\Sigma$-superpolynomials.

We define the set of $\Sigma$-superidentities of any $\Sigma$-superalgebra $\mathfrak{A}$ as the intersection of all kernels of all grading-preserving $C[\varepsilon]$-homomorphisms $\phi: C[\varepsilon]\left\langle X^{(g)}\right\rangle \rightarrow \mathfrak{A}$, and denote it by $\operatorname{id}_{\Sigma}(\mathfrak{A})$.

Definition 4.9. For every finitely supported function $\bar{n}: 2^{<\omega} \rightarrow \mathbb{N}, g \mapsto \bar{n}^{(g)}$, we let $P_{\bar{n}}[\varepsilon]$ denote the $C[\varepsilon]$-module of multilinear $\Sigma$-superpolynomials with coefficients in $C[\varepsilon]$, in the variables $\left\{x_{i}^{(g)}\right\}_{1 \leq i \leq \bar{n}^{(g)}, g \in 2^{<\omega}}$. We will refer to $\bar{n}$ as the associated multidegree. We will also write $n=\sum \bar{n}^{(g)}$, the total degree of identities in $P_{\bar{n}}[\varepsilon]$. The multilinear part of $C[\varepsilon]\left\langle X^{(g)}\right\rangle$ is $\bigoplus_{\bar{n}} P_{\bar{n}}[\varepsilon]$.

Again, keeping the analogy to the case of characteristic 0, we can define the operation of the generalized Grassmann hull on an identity.

Definition 4.10. We define the Grassmann involution on $\Sigma$-superpolynomials as follows. Let $f=\sum_{\sigma \in S_{n}} a_{\sigma} x_{\sigma(1)} \cdots x_{\sigma(n)} \in P_{\bar{n}}[\varepsilon]$ be a multilinear $2^{<\omega_{\text {-graded }}}$ 
identity of multidegree $\bar{n}$, such that each variable $x_{j}$ is in the homogenous component of $C[\varepsilon]\left\langle X^{(g)}\right\rangle$ corresponding to some $g_{j}$. Then

$$
f^{*}=\sum_{\sigma \in S_{n}} \mathfrak{s g n}_{w}(\sigma) a_{\sigma} x_{\sigma(1)} \cdots x_{\sigma(n)},
$$

where $w=\left(e_{g_{1}}, \ldots, e_{g_{n}}\right)$.

This is indeed an involution:

Lemma 4.11. The map $f \mapsto f^{*}$ is an involution.

Proof. Let $f \in P_{\bar{n}}[\varepsilon]$ be a multilinear $2^{<\omega}$-graded identity of multidegree $\bar{n}$. Write $f=\sum_{\sigma \in S_{n}} a_{\sigma} x_{\sigma(1)} \cdots x_{\sigma(n)}$. Then $f^{* *}=\sum_{\sigma \in S_{n}} \mathfrak{s g n}_{w}(\sigma)^{2} a_{\sigma} x_{\sigma(1)} \cdots x_{\sigma(n)}$. But,

$$
\mathfrak{s g n}_{w}(\sigma)^{2}=\exp \left(\sum_{\substack{i<j \\ \sigma(i)>\sigma(j)}} \varepsilon_{w_{\sigma(i)}} \varepsilon_{w_{\sigma(j)}}\right)^{2}=1
$$

by Remark 3.20, so $f^{* *}=f$.

As is the case with superalgebras, the involution gives the identities of the generalized Grassmann hull:

Definition 4.12. Let $\Delta \triangleleft C[\varepsilon]\left\langle X^{(g)}\right\rangle$ be a two-sided ideal. We say that $\Delta$ is a $\mathrm{T}_{\Sigma}$-ideal if it is also invariant under all $C[\varepsilon]$-endomorphisms of $C[\varepsilon]\left\langle X^{(g)}\right\rangle$ that preserve the grading.

Also, in this case, we let $\Delta^{*}$ be the $\mathrm{T}_{\Sigma}$-ideal generated as a $\mathrm{T}_{\Sigma}$-ideal by the images of all multilinear identities in $\Delta$ under the involution $*$.

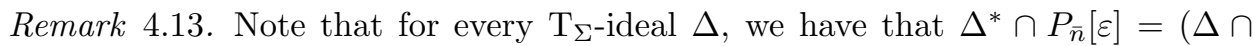
$\left.P_{\bar{n}}[\varepsilon]\right)^{*}$, where on the right hand side, taking $*$ means taking $*$ on each element separately. This is because the multilinear part $\Delta \cap P_{\bar{n}}[\varepsilon]$ is already endomorphisminvariant, and since by definition $\Delta^{*}$ is the minimal $\mathrm{T}_{\Sigma}$-ideal containing $\left(\Delta \cap P_{\bar{n}}[\varepsilon]\right)^{*}$.

In other words, using $*$ on all multilinear identities of a $\mathrm{T}_{\Sigma}$-ideal $\Delta$ gives all multilinear identities of $\Delta^{*}$.

Recall that $\operatorname{id}_{\Sigma}(\mathfrak{A})$ is the set of $\Sigma$-superidentities of $\mathfrak{A}$, Definition 4.8 .

Theorem 4.14. Let $\mathfrak{A}$ be a $\Sigma$-superalgebra. Then $\operatorname{id}_{\Sigma}(\mathfrak{S}[\mathfrak{A}])$ and $\operatorname{id}_{\Sigma}(\mathfrak{A})^{*}$ have the same multilinear components.

In other words, for every $f \in P_{\bar{n}}[\varepsilon]$, we have that $f \in \operatorname{id}_{\Sigma}(\mathfrak{S}[\mathfrak{A}])$ if and only if $f^{*} \in \operatorname{id}_{\Sigma}(\mathfrak{A})$.

Proof. Let $f=\sum_{\sigma \in S_{n}} \alpha_{\sigma} x_{\sigma(1)} \cdots x_{\sigma(n)} \in P_{\bar{n}}[\varepsilon]$. Let $x_{i} \mapsto a_{i} \otimes w_{i}$ be any substitution where $w_{i} \in \mathfrak{S}_{g_{i}}$ is a word in the generators $e_{j}^{(n)}$ of $\mathfrak{S}$, in the component 
corresponding to $g_{i}$, and $a_{i} \in \mathfrak{A}_{g_{i}}$. Then, under the substitution:

$$
\begin{aligned}
& f \quad \mapsto \quad \sum_{\sigma \in S_{n}} \alpha_{\sigma} a_{\sigma(1)} \otimes w_{\sigma(1)} \cdots a_{\sigma(n)} \otimes w_{\sigma(n)} \\
& =\quad \sum_{\sigma \in S_{n}} \alpha_{\sigma}\left(a_{\sigma(1)} \cdots a_{\sigma(n)}\right) \otimes\left(w_{\sigma(1)} \cdots w_{\sigma(n)}\right) \\
& \text { Prop. }=3.23 \sum_{\sigma \in S_{n}} \alpha_{\sigma}\left(a_{\sigma(1)} \cdots a_{\sigma(n)}\right) \otimes\left(\mathfrak{s g n}_{w}(\sigma) w_{1} \cdots w_{n}\right) \\
& =\quad \sum_{\sigma \in S_{n}} \alpha_{\sigma}\left(\mathfrak{s g n}_{w}(\sigma) a_{\sigma(1)} \cdots a_{\sigma(n)}\right) \otimes\left(w_{1} \cdots w_{n}\right) \\
& =\left(\sum_{\sigma \in S_{n}} \alpha_{\sigma} \mathfrak{s g \mathfrak { n }} w(\sigma) a_{\sigma(1)} \cdots a_{\sigma(n)}\right) \otimes\left(w_{1} \cdots w_{n}\right) \\
& =f^{*}\left(a_{1}, \ldots, a_{n}\right) \otimes w_{1} \cdots w_{n},
\end{aligned}
$$

as we wanted to show.

We say that $\Sigma$-superalgebras $\mathfrak{A}$ and $\mathfrak{B}$ are multilinearly equivalent if $\operatorname{id}_{\Sigma}(\mathfrak{A})$ and $\operatorname{id}_{\Sigma}(\mathfrak{B})$ share the same multiliner identities.

Corollary 4.15. For every $\Sigma$-superalgebra $\mathfrak{A}$, $\mathfrak{S}[\mathfrak{S}[\mathfrak{A}]]$ is multilinearly equivalent to $\mathfrak{A}$.

Proof. Use the result of Theorem 4.14 twice, and then apply Lemma 4.11

Remark 4.16. We have not proved that $\operatorname{id}_{\Sigma}(\mathfrak{S}[\mathfrak{A}])=\operatorname{id}_{\Sigma}(\mathfrak{A})^{*}$. In characteristic 0 , having the same multilinear identities would have implied that they are the same. However, this is not the case in positive characteristic: $\operatorname{id}_{\Sigma}(\mathfrak{A})$ is not necessarily generated as a $\mathrm{T}_{\Sigma}$-ideal by its multilinear component.

We see that even though the language of generalized Grassmann hulls generalizes the ordinary notion of Grassmann hull, its formulation could be considered more elegant; rather than defining the involution on a multilinear identity by multiplying by the sign of only the odd variables, we simply multiply by the generalized sign of all variables. This is mainly because all words in the generators $e_{i}$ of $\mathfrak{G}$ are, in a way, generic, so no special treatment is needed for any specific component of the grading.

\section{Generalized supertraces}

The superization of basic concepts in linear algebra, such as the supertrace and supercommutator, is defined in characteristic zero. We now begin the development of a supertheory based upon $\mathfrak{G}$ and the concept of the generalized superalgebra. Such a $\Sigma$-supertheory will have the advantage of being characteristic free, valid over any ring.

We will begin by defining the notion of $\Sigma$-supertraces. Recall that an (abstract) trace function on a $C$-algebra $A$ is a function $\operatorname{tr}: A \rightarrow \operatorname{Cent}(A)$ satisfying for any $a, b \in A$ the conditions

$$
\operatorname{tr}[a, b]=0
$$


and

$$
\operatorname{tr}(a \operatorname{tr}(b))=\operatorname{tr}(a) \operatorname{tr}(b) .
$$

Definition 5.1. Let $\mathfrak{A}$ be a $\Sigma$-superalgebra over $C$. Its $\Sigma$-supercenter, $\mathfrak{s} \mathfrak{C} \mathfrak{n} \mathfrak{t}(\mathfrak{A})$, is the set of all elements of $\mathfrak{A}$ that $\Sigma$-supercommute with every element, i.e.

$$
\mathfrak{s C e n t}(\mathfrak{A})=\{a \in \mathfrak{A} \mid \forall b \in \mathfrak{A},\{a, b\}=0\},
$$

where $\{a, b\}$ is the $\Sigma$-supercommutator of Definition 4.3 .

Definition 5.2. Let $\mathfrak{A}$ be a $\Sigma$-superalgebra over $C$. A $C[\varepsilon]$-linear (gradingpreserving) function $\mathfrak{s t r}: \mathfrak{A} \rightarrow \mathfrak{s C e n t}(\mathfrak{A})$ will be called a $\Sigma$-supertrace if

$$
\mathfrak{s t r}\{a, b\}=0
$$

and

$$
\mathfrak{s t r}(a \mathfrak{s t r}(b))=\mathfrak{s t r}(a) \mathfrak{s t r}(b),
$$

for every $a, b \in \mathfrak{A}$.

The concepts of $\Sigma$-supertrace $\Sigma$-superidentities naturally follows (see BR05, Chapter 12]).

Definition 5.3. Define the algebra $C[\varepsilon]\left\langle X^{(g)}, \mathfrak{s T r}\right\rangle$ to be the free $\Sigma$-superalgebra with $\Sigma$-supertrace $\mathfrak{s} \mathfrak{T r}$. This algebra is spanned over $C[\varepsilon]$ by words of the form $w_{0} \mathfrak{s} \mathfrak{T} \mathfrak{r}\left(w_{1}\right) \cdots \mathfrak{s} \mathfrak{T} \mathfrak{r}\left(w_{\ell}\right)$ where $w_{i} \in\left\langle X^{(g)}\right\rangle$, and the grading is such that the grade of $\mathfrak{s T r}(w)$ is the same as that of $w$. The defining relations are the axioms of Definition 5.2 .

The $\Sigma$-supertrace $\Sigma$-superidentities of a $\Sigma$-superalgebra $\mathfrak{A}$ with $\Sigma$-supertrace $\mathfrak{s t r}$ are the elements in the intersection of all the kernels of all grading-preserving $C[\varepsilon]$ homomorphisms $\phi: C[\varepsilon]\left\langle X^{(g)}, \mathfrak{s} \mathfrak{T r}\right\rangle \rightarrow A$ such that $\mathfrak{s t r} \phi(x)=\phi(\mathfrak{s} \mathfrak{T r} x)$.

Remark 5.4. We use different capitalization to differentiate between formal traces (traces in the free algebra) and traces of the object under discussion. That is, $\operatorname{Tr}, \mathbf{s T r}$ and $\mathfrak{s} \mathfrak{T r}$ are formal traces, formal supertraces and formal $\Sigma$-supertraces in the algebras $C\langle X, \operatorname{Tr}\rangle, C\left\langle X^{(0)}, X^{(1)}, \mathrm{sTr}\right\rangle$ and $C[\varepsilon]\left\langle X^{(g)}, \mathfrak{s} \mathfrak{T r}\right\rangle$, respectively. At the same time, tr, str and $\mathfrak{s t r}$ are arbitrary trace functions, in any algebra we happen to be currently working with.

For example, the equality $\mathfrak{s t r}\left(a^{p}\right)=\mathfrak{s t r}(a)^{p}$ holds in the algebra $A$ for all $a$, if and only if $A$ satisfies the $\Sigma$-supertrace $\Sigma$-superidentity $\mathfrak{s T r}\left(x^{p}\right)=\mathfrak{s T r}(x)^{p}$. In other words, $\mathfrak{s} \mathfrak{T} \mathfrak{r}\left(x^{p}\right)=\mathfrak{s} \mathfrak{T r}(x)^{p}$ is an identity, while $\mathfrak{s t r}\left(a^{p}\right)=\mathfrak{s t r}(a)^{p}$ is the value of that identity after substituting the function $\mathfrak{s t r}$ to the variable $\mathfrak{s} \mathfrak{T r}$.

We come to our most important example.

Definition 5.5. Let $\mathfrak{A}$ be a $\Sigma$-superalgebra with a grading preserving trace function $\operatorname{tr}: \mathfrak{A} \rightarrow C$. Define the associated $\Sigma$-supertrace function $\mathfrak{s t r}=\operatorname{tr}^{*}$ on $\mathfrak{S}[\mathfrak{A}]$ by $\mathfrak{s t r}(a \otimes w)=\operatorname{tr}(a) \otimes w$.

Conversely, if $\mathfrak{A}$ has a $\Sigma$-supertrace $\mathfrak{s t r}$, define its associated trace function $\operatorname{tr}=$ $\mathfrak{s t r}{ }^{*}$ on $\mathfrak{S}[\mathfrak{A}]$ by $\operatorname{tr}(a \otimes w)=\mathfrak{s t r}(a) \otimes w$. Note that $\mathfrak{s t r}^{*}$ preserves the grading.

Lemma 5.6. The above definitions of the associated trace function $\mathfrak{s t r}^{*}$ and the associated $\Sigma$-supertrace function $\mathrm{tr}^{*}$ indeed give a trace function and a $\Sigma$-supertrace function, respectively. 
Proof. This follows since for all $a \otimes u, b \otimes v \in \mathfrak{S}[\mathfrak{A}], a, b \in \mathfrak{A}, u, v \in \mathfrak{S}$,

$$
\begin{aligned}
\{a \otimes u, b \otimes v\} & =(a \otimes u)(b \otimes v)-\exp \left(\varepsilon_{g} \varepsilon_{h}\right)(b \otimes v)(a \otimes u) \\
& =a b \otimes u v-b a \otimes \exp \left(\varepsilon_{g} \varepsilon_{h}\right) v u \\
& =(a b-b a) \otimes u v=[a, b] \otimes u v
\end{aligned}
$$

and $\{a, b\} \otimes u v=[a \otimes u, b \otimes v]$ in the same manner.

Remark 5.7. Let $A$ be a $C$-algebra, with a trace function tr. Then the algebra $A \otimes_{C}$ $C[\varepsilon]\left[2^{<\omega}\right]$ has a $\Sigma$-superalgebra grading (coming from the grading of the $C[\varepsilon]\left[2^{<\omega}\right]$ component), and is a $\Sigma$-superalgebra. Now, the function $\operatorname{tr}: A \rightarrow \operatorname{Cent}(A)$ can be extended by linearity to $\hat{\operatorname{tr}}=\operatorname{tr} \otimes 1: A \otimes_{C} C[\varepsilon]\left[2^{<\omega}\right] \rightarrow \operatorname{Cent}(A) \otimes C[\varepsilon]\left[2^{<\omega}\right]$ such that

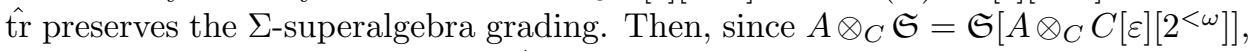
we obtain a $\Sigma$-supertrace $\mathfrak{s t r}=\hat{\operatorname{tr}}^{*}$ on $A \otimes_{C} \mathfrak{S}$, given by: $\mathfrak{s t r}(a \otimes w)=\operatorname{tr}(a) \otimes w$. This construction generalizes Definition 5.5 to the case of (non-graded) $C$-algebras.

Now, analogously to Theorem 3.14, we show the equivalence of supertrace and $\Sigma$-supertrace identities (the identities are not graded, so these are not $\Sigma$-superidentities).

Theorem 5.8. Suppose that 2 is invertible in $C$. Let $A$ be some $C$-algebra with trace tr. Let $\mathfrak{s t r}$ be the associated $\Sigma$-supertrace of $A \otimes_{C} \mathfrak{S}$, and in a similar manner, associate a supertrace $\operatorname{str}$ to $A \otimes_{C} S$, where $S$ is the free supercommutative algebra. Then the supertrace identities of $A \otimes_{C} S$ are the same as the $\Sigma$ supertrace identities of $A \otimes_{C} \mathfrak{S}$, with $\mathfrak{s} \mathfrak{T r}$ replaced by $\mathrm{sTr}$.

Proof. The proof is virtually identical, word for word, to the proof of Theorem 3.14

A key result in PI-theory is the "Kemer supertrick" (see e.g. [Zel91]), which heavily relies on representation theory, which fails to deliver in positive characteristic. The Kemer supertrick can be reformulated as the claim that for every algebra $A$ there is some $n$ such that $\operatorname{id}(A) \supseteq \operatorname{id}\left(\mathrm{M}_{n}(G)\right)$. In this sense, the Kemer supertrick has already been proven in characteristic $p$ (by Kemer, Kem95]), but with very bad bounds.

Eventually, one might hope to bypass this difficulty by directly adding formal supertraces to algebras (and then show that their identities imply all identities of $\mathrm{M}_{n}(\mathfrak{G})$ ), just like Zubrilin's theory enables the introduction of traces to an algebra and showing that affine PI-algebras satisfy all identities of a matrix algebra (see AB10, for an overview of Zubrillin traces).

This motivates the following question about $\Sigma$-supertraces:

Question 5.9. Let $A$ be an (ordinary) algebra on which a linear function $\mathfrak{f}$ is defined. What identities on $A$ and $\mathfrak{f}$ allow us to introduce a grading to the algebra such that $\mathfrak{f}$ becomes a $\Sigma$-supertrace?

More formally, we define

Definition 5.10. Let $C\langle X, \mathfrak{F}\rangle$ be the free algebra over $C$ with a $C$-linear function $\mathfrak{F}$ acting freely on it. Let $A$ be any $C$-algebra with a linear function $\mathfrak{f}: A \rightarrow A$. 
We define the identities of $A$ with linear function $\mathfrak{f}$ to be the intersection of all kernels of all homomorphisms $\phi: C\langle X, \mathfrak{F}\rangle \rightarrow A$ such that $\phi(\mathfrak{F}(a))=\mathfrak{f}(\phi(a))$.

Remark 5.11. As in Remark 5.4, we use capitalization to differentiate formal objects from others. That is, $\mathfrak{f}$ is any particular linear function, while $\mathfrak{F}$ is the formal linear function, of the algebra $C\langle X, \mathfrak{F}\rangle$.

Theorem 5.12. The multilinear part of the ideal of identities of $C[\varepsilon]\left\langle X^{(g)}, \mathfrak{s} \mathfrak{T r}\right\rangle$ with linear function $\mathfrak{s} \mathfrak{T r}$ is generated by:

$$
\begin{aligned}
\mathfrak{F}(\mathfrak{F}(x) y) & =\mathfrak{F}(x) \mathfrak{F}(y) \\
\mathfrak{F}(x \mathfrak{F}(y)) & =\mathfrak{F}(x) \mathfrak{F}(y) \\
{[x, \mathfrak{F}[y, z]] } & =0 \\
{[\mathfrak{F}(x),[\mathfrak{F}(y), z]] } & =0
\end{aligned}
$$

Note that the $\Sigma$-superidentity $\mathfrak{F}\{a, b\}=0$ of Definition 5.2 is not in the list, as it is not an (ordinary) identity.

To prove the theorem we require a few lemmas. We begin by proving a lemma analogous to Lemma 3.8

Lemma 5.13. The following identities with linear function hold in $C[\varepsilon]\left\langle X^{(g)}, \mathfrak{s T r}\right\rangle$ :

$$
\begin{aligned}
\mathfrak{F}(\mathfrak{F}(x) y) & =\mathfrak{F}(x) \mathfrak{F}(y) \\
\mathfrak{F}(x \mathfrak{F}(y)) & =\mathfrak{F}(x) \mathfrak{F}(y) \\
{[x, \mathfrak{F}[y, z]] } & =0 \\
{[\mathfrak{F}(x),[\mathfrak{F}(y), z]] } & =0
\end{aligned}
$$

Proof. The identities (7a) and (7b) follow immediately from the definition of the $\Sigma$-supertrace (Definition 5.2).

We will now show that the identities $(7 \mathrm{c})$ and $(7 \mathrm{~d})$ are indeed satisfied by any $\Sigma$-supertrace, using the fact that the $\Sigma$-supertraces $\Sigma$-supercommute with everything and a product of two elements inside a $\Sigma$-supertrace behaves as if it $\Sigma$ supercommutes. Thus, for the purpose of checking (7c) and (7d), one can assume that everything $\Sigma$-supercommutes. But the $\Sigma$-supercommutative $\Sigma$-superalgebra $\mathfrak{G}$ satisfies the Grassmann identity, which thus implies these two identities.

More formally, we begin by proving (7c). The proof of $(7 \mathrm{~d})$ is completely analogous. First of all, since (7c) is multilinear, we may assume that $x, y$ and $z$ are all homogenous. Then the following holds:

$$
\begin{aligned}
{[x, \mathfrak{s T r}[y, z]] } & =[x, \mathfrak{s T r}(y z)-\mathfrak{s} \mathfrak{T r}(z y)]=\left[x, \mathfrak{s T r}(y z)-\exp \left(\varepsilon_{y} \varepsilon_{z}\right) \mathfrak{s} \mathfrak{T r}(y z)\right] \\
& =\left(1-\exp \left(\varepsilon_{y} \varepsilon_{z}\right)\right)[x, \mathfrak{s T r}(y z)] \\
& =\left(1-\exp \left(\varepsilon_{y} \varepsilon_{z}\right)\right)(x \mathfrak{s} \mathfrak{T r}(y z)-\mathfrak{s} \mathfrak{T} \mathfrak{r}(y z) x) \\
& =\left(1-\exp \left(\varepsilon_{y} \varepsilon_{z}\right)\right)\left(x \mathfrak{s} \mathfrak{T} \mathfrak{r}(y z)-\exp \left(\varepsilon_{x} \varepsilon_{y z}\right) x \mathfrak{s} \mathfrak{T r}(y z)\right) \\
& =\left(1-\exp \left(\varepsilon_{y} \varepsilon_{z}\right)\right)\left(1-\exp \left(\varepsilon_{x}\left(\varepsilon_{y}+\varepsilon_{z}\right)\right)\right) x \mathfrak{s} \mathfrak{T}(y z) .
\end{aligned}
$$

Hence, in order to show that this is zero, it is sufficient to show that

$$
0=\left(1-\exp \left(\varepsilon_{y} \varepsilon_{z}\right)\right)\left(1-\exp \left(\varepsilon_{x}\left(\varepsilon_{y}+\varepsilon_{z}\right)\right)\right) .
$$


However, if we choose words $w_{x}, w_{y}, w_{z} \in \mathfrak{G}$ such that $\varepsilon_{w_{x}}=\varepsilon_{x}, \varepsilon_{w_{y}}=\varepsilon_{y}$ and $\varepsilon_{w_{z}}=\varepsilon_{z}$, then $\mathfrak{G}$ satisfies the Grassmann identity

$$
\begin{aligned}
0=\left[w_{x},\left[w_{y}, w_{z}\right]\right] & =\left[w_{x}, w_{y} w_{z}-w_{z} w_{y}\right]=\left[w_{x}, w_{y} w_{z}-\exp \left(\varepsilon_{y} \varepsilon_{z}\right) w_{y} w_{z}\right] \\
& =\left(1-\exp \left(\varepsilon_{y} \varepsilon_{z}\right)\right)\left[w_{x}, w_{y} w_{z}\right] \\
& =\left(1-\exp \left(\varepsilon_{y} \varepsilon_{z}\right)\right)\left(w_{x} w_{y} w_{z}-w_{y} w_{z} w_{x}\right) \\
& =\left(1-\exp \left(\varepsilon_{y} \varepsilon_{z}\right)\right)\left(w_{x} w_{y} w_{z}-\exp \left(\varepsilon_{x} \varepsilon_{y z}\right) w_{x} w_{y} w_{z}\right) \\
& =\left(1-\exp \left(\varepsilon_{y} \varepsilon_{z}\right)\right)\left(1-\exp \left(\varepsilon_{x}\left(\varepsilon_{y}+\varepsilon_{z}\right)\right)\right) w_{x} w_{y} w_{z},
\end{aligned}
$$

and thus $\left(1-\exp \left(\varepsilon_{y} \varepsilon_{z}\right)\right)\left(1-\exp \left(\varepsilon_{x}\left(\varepsilon_{y}+\varepsilon_{z}\right)\right)\right)=0$, as we wanted to show.

Now we state a fact analogous to Lemma 3.9

Lemma 5.14. The identities with linear function:

$$
\begin{aligned}
{[x,[\mathfrak{F}(y), \mathfrak{F}(z)]] } & =0 \\
{[x, \mathfrak{F}(y)][\mathfrak{F}(z), \mathfrak{F}(w)]+[x, \mathfrak{F}(z)][\mathfrak{F}(y), \mathfrak{F}(w)] } & =0 \\
{[\mathfrak{F}(x), y][\mathfrak{F}(z), \mathfrak{F}(w)]+[\mathfrak{F}(x), \mathfrak{F}(z)][y, \mathfrak{F}(w)] } & =0
\end{aligned}
$$

are consequences of $(7 \mathrm{a}),(7 \mathrm{~b}),(7 \mathrm{c})$ and $(7 \mathrm{~d})$.

Proof. In order to obtain (8a), substitute $y \mapsto \mathfrak{F}(y)$ into (7c), and use (7a) and $(7 \mathrm{~b})$ to see that $0=[x, \mathfrak{F}[y, z]] \mapsto[x, \mathfrak{F}[\mathfrak{F}(y), z]]=[x,[\mathfrak{F}(y), \mathfrak{F}(z)]]$. The proofs of $(8 \mathrm{~b})$ and (8c), given (17d ) and (8a) are completely analogous to the proof of Lemma 3.9 .

Proof of Theorem 5.12, We will use the following equalities: for all words $s=$ $x_{1} \cdots x_{n}$ and $t$, we have

(9a) $[s, t]=\left[x_{1}, x_{2} \cdots x_{n} t\right]+\left[x_{2}, x_{3} \cdots x_{n} t x_{1}\right]+\cdots+\left[x_{n}, t x_{1} x_{2} \cdots x_{n-1}\right]$,

(9b) $\quad 0=\left[x_{1}, x_{2} \cdots x_{n}\right]+\left[x_{2}, x_{3} \cdots x_{n} x_{1}\right]+\cdots+\left[x_{n}, x_{1} x_{2} \cdots x_{n-1}\right]$.

The strategy of our proof greatly resembles that of Lemma 3.10. We will use the above identities to bring an arbitrary polynomial $f \in C\langle X, \mathfrak{F}\rangle$ to a specified standard form, and then use substitutions to show that the coefficients are 0 . This will be done via substitutions from the matrix algebras $\mathrm{M}_{n}(\mathfrak{G})$ over $\mathfrak{G}$, with the $\Sigma$-supertraces $\mathfrak{s t r}$ associated with the usual traces in $\mathrm{M}_{n}(C)$.

We begin by specifying the standard form we will use. Note that we are working with multilinear polynomials. The form is a sum of terms of the form:

$$
\begin{gathered}
w \times \\
\mathfrak{F}\left(v_{1}\right) \mathfrak{F}\left(v_{2}\right) \cdots \mathfrak{F}\left(v_{n}\right) \times \\
{\left[w_{1}, \mathfrak{F}\left(u_{1}\right)\right]\left[w_{2}, \mathfrak{F}\left(u_{2}\right)\right] \cdots\left[w_{m}, \mathfrak{F}\left(u_{m}\right)\right] \times} \\
{\left[\mathfrak{F}\left(u_{m+1}\right), \mathfrak{F}\left(u_{m+2}\right)\right]\left[\mathfrak{F}\left(u_{m+3}\right), \mathfrak{F}\left(u_{m+4}\right)\right] \cdots\left[\mathfrak{F}\left(u_{k-1}\right), \mathfrak{F}\left(u_{k}\right)\right] \times} \\
\mathfrak{F}\left[s_{1}, t_{1}\right] \mathfrak{F}\left[s_{2}, t_{2}\right] \cdots \mathfrak{F}\left[s_{\ell}, t_{\ell}\right]
\end{gathered}
$$

where $w, w_{1}, \ldots, w_{m}, v_{1}, \ldots, v_{n}, u_{1}, \ldots, u_{k}$ and $t_{1}, \ldots, t_{\ell}$ are all words in the $x_{i}$, and the $s_{1}, \ldots, s_{\ell}$ are letters. However, many of these forms are trivially equal, so we require that: the words $u_{1}, \ldots, u_{k}$ are alphabetically ordered; the words $v_{1}, \ldots, v_{n}$ are alphabetically ordered; the pairs $\left(s_{i}, t_{i}\right)$ are also alphabetically ordered; for every $i$, the letter $s_{i}$ is smaller than some letter of $t_{i}$; and the words $v_{i}$ and $u_{i}$ are cyclically minimal, where a word is cyclically minimal if it is the first among its cyclic rotations. 
Lemma 5.13, Lemma 5.14 and (9a), (9b) imply that every multilinear polynomial can be brought to this form.

Now, we will show that the coefficients of the terms containing no $\mathfrak{F}$ 's are zero. Indeed, substitute matrix units $x_{i} \mapsto e_{\sigma(i), \sigma(i+1)}$ into all $x_{i}$, where $\sigma$ is some permutation. Then only the monomial in which the $x_{i}$ are ordered according to $\sigma$ contributes, and thus its coefficient is 0 .

Next, rather than substitute a path as we just did, we choose some subset of the variables and substitute a cycle into them and a path into the rest. Since the standard trace is zero off-diagonally, the only terms contributing are those that have no more than one appearance of $\mathfrak{F}$, corresponding to the cycle. We thus have three options for the terms that contribute: $w \cdot \mathfrak{F}\left(v_{1}\right), w \cdot\left[w_{1}, \mathfrak{F}\left(u_{1}\right)\right]$ and $w \cdot \mathfrak{F}\left[s_{1}, t_{1}\right]$.

Note that the last two do not contribute at all if the coefficients of the matrix units are central. Thus the coefficient of the first is 0 . Now, substitute coefficients from $\mathfrak{G}$ to two edges of the loop, such that exactly one edge has $e_{1}$ as the coefficient, and another has $e_{2}$ as the coefficient. Then only the term $w \cdot \mathfrak{F}\left[s_{1}, t_{1}\right]$ contributes - and hence has coefficient equal to 0 . Finally, substitute $e_{1}$ to just one of the variables of the loop, and $e_{2}$ to an edge of the path. Then the term $w \cdot\left[w_{1}, \mathfrak{F}\left(u_{1}\right)\right]$ gives a non-zero contribution, unless it too has coefficient zero.

We use induction on $N=n+k+\ell$ to show that all coefficients are 0 . We substitute matrix elements such that there is one path, and $N=n+k+\ell$ loops. We are now left with the liberty to choose their coefficients from $\mathfrak{G}$. Now, we must be able to tell how they are partitioned into $v_{i}$ 's, $u_{i}$ 's and $\left(s_{i}, t_{i}\right)$ 's. So, at first we substitute only central coefficients. This gives us the case of: $k=\ell=0$, so its coefficient is zero.

Now, we will use induction on $k+\ell$. We choose $n=N-(k+\ell)$ loops, and substitute central coefficients. This forces them to be $v_{1}, \ldots, v_{n}$, and by induction, no coefficient with any other $v_{i}$ 's contributes. Now, we substitute coefficients $e_{i}$ into all elements of the path, and we substitute one coefficient into the generators in each remaining loop (out of the $k+\ell$ loops left). This gives us the case where $\ell=0$.

We use induction on $\ell$. Choose $k$ loops, and substitute one coefficient into each one of them, in addition to the substitution into elements of the path. This forces these loops to be the $u_{1}, \ldots, u_{k}$. We are left with two things to find out: how is the path split into the $w, w_{1}, \ldots, w_{m}$, and how are the remaining $\ell$ loops partitioned between the $s_{i}$ and the $t_{i}$.

Choosing the partition of each remaining loop into $s_{i}$ and $t_{i}$ is easy, and will be done via induction on the position of the letter $s_{i}$ relative to the largest letter of $t_{i}$. Indeed, the base of the induction is this: substitute a coefficient $e_{i_{1}}$ to the largest letter and $e_{i_{2}}$ to the letter before it. Then the only contribution to the coefficient of the product $\varepsilon_{i_{1}} \varepsilon_{i_{2}}$ comes from the cases in which the largest letter itself is $s_{i}$, or the one before it is $s_{i}$ (otherwise $e_{i_{1}}$ and $e_{i_{2}}$ appear in their correct order). But because the largest letter is never $s_{i}$, we see that $s_{i}$ is also never the letter before that. Proceeding by induction, we are done.

Therefore, we have almost isolated all coefficients of the form; we must now isolate one specific way to break down the path to $w, w_{1}, \ldots, w_{m}$, for an arbitrary (but known) choice of $s_{i}$. This is done as follows. We use induction on $m$. Now, we already know that the associated loop, $u_{i}$, has one coefficient, say $e_{1}$, and we know which loop it is. Also recall that we substituted coefficients into the elements of 
the path. So, after the substitution, look for the largest number of $\varepsilon_{i}$ 's appearing. This information determines which elements of the path belong to $w$ (their $\varepsilon_{i}$ 's never appear). Now look for the smallest number of $\varepsilon_{i}$ 's from the path appearing. This is the case where each $w_{i}$ contributes one $\varepsilon_{i}$. So, sort these $\varepsilon_{i}$ 's, and put the element of the path corresponding to the $j$-th $\varepsilon_{i}$ into $w_{j}$. This gives us all elements of $w_{j}$, and only the case where $m$ is the smallest value we have not considered, contributes.

This isolates everything - only one term contributes, and thus has a coefficient of zero, which completes all of the above inductive steps.

Note that incidently, just like in Lemma 3.10 we also obtain the co-dimension sequence.

Corollary 5.15. Suppose that $A$ is any $C$-algebra, and $\mathfrak{f}$ any linear function on it. Also suppose that the following is true in A:

$$
\begin{aligned}
\mathfrak{f}(\mathfrak{f}(x) y) & =\mathfrak{f}(x) \mathfrak{f}(y), \\
\mathfrak{f}(x \mathfrak{f}(y)) & =\mathfrak{f}(x) \mathfrak{f}(y), \\
{[x, \mathfrak{f}[y, z]] } & =0, \\
{[\mathfrak{f}(x),[\mathfrak{f}(y), z]] } & =0 .
\end{aligned}
$$

Then there is some $\Sigma$-superalgebra $\mathfrak{A}$ with $\Sigma$-supertrace $\mathfrak{s t r}$, such that $A$ and $\mathfrak{A}$ have the same multilinear identities with linear function $\mathfrak{f}$ and $\mathfrak{s t r}$ respectively.

5.1. Concluding remarks. We have seen how the structure of the generalized Grassmann algebra can be used to generalize the notions of superalgebras and supertraces to arbitrary characteristics and rings. In a similar manner, one can define a Lie $\Sigma$-superalgebra:

Definition 5.16. Let $\mathfrak{L}$ be a $C[\varepsilon]$-module with a $\Sigma$-superalgebra grading. Suppose that $\{\cdot, \cdot\}$ is a bi-linear form that respects the grading (if $a \in \mathfrak{L}_{g}, b \in \mathfrak{L}_{h}$ then $\{a, b\} \in \mathfrak{L}_{g h}$ ). Then $\mathfrak{L}$ will be called a Lie $\Sigma$-superalgebra if for every homogenous $x, y, z \in \mathfrak{L}:$

(1) $\{x, y\}=-\exp \left(\varepsilon_{x} \varepsilon_{y}\right)\{y, x\}$,

(2) $\exp \left(\varepsilon_{x} \varepsilon_{z}\right)\{x,\{y, z\}\}+\exp \left(\varepsilon_{y} \varepsilon_{x}\right)\{y,\{z, x\}\}+\exp \left(\varepsilon_{z} \varepsilon_{y}\right)\{z,\{x, y\}\}=0$,

(3) $\{x,\{x, x\}\}=0$.

Note that (3) is superfluous when 3 is invertible in $C$. This new object is obviously equivalent to an ordinary Lie superalgebra whenever 2 is invertible. However, the interesting property of this definition is that it yields non-trivial behavior in characteristic 2, where (unlike ordinary Lie superalgebras) it does not degenerate to an ordinary Lie algebra.

In this paper we only considered $\Sigma$-supertheory from the point of view of PItheory. In a similar manner, one can consider all of $\Sigma$-supertheory in characteristic 2. The cost we pay for this is that since the grading is over an infinite group, we must consider infinite-dimensional objects; therefore, in order to replicate the study of finite dimensional objects, one should consider $\Sigma$-superobjects that are locally finite-dimensional, in the sense that their graded components are each finite dimensional and isomorphic to one another in a sufficiently strong sense (so infinite-dimensional behavior is not "hidden" across multiple graded components).

One hopes that this construction can be used to yield characteristic-free results over arbitrary rings, such as Theorem 5.12 . 


\section{REFERENCES}

[AB10] Eli Aljadeff and Alexei Kanel-Belov, Representability and Specht problem for G-graded algebras, Adv. Math. 225 (2010), no. 5, 2391-2428, DOI 10.1016/j.aim.2010.04.025. MR2680170

[Bel00] A. Kanel-Belov, Counterexamples to the Specht problem (Russian, with Russian summary), Mat. Sb. 191 (2000), no. 3, 13-24, DOI 10.1070/SM2000v191n03ABEH000460; English transl., Sb. Math. 191 (2000), no. 3-4, 329-340. MR.1773251

[BR05] Alexei Kanel-Belov and Louis Halle Rowen, Computational aspects of polynomial identities, Research Notes in Mathematics, vol. 9, A K Peters, Ltd., Wellesley, MA, 2005. MR2124127

[DM99] Pierre Deligne and John W. Morgan, Notes on supersymmetry (following Joseph Bernstein), Quantum fields and strings: a course for mathematicians, Vol. 1, 2 (Princeton, NJ, 1996/1997), Amer. Math. Soc., Providence, RI, 1999, pp. 41-97. MR1701597

[GK01] Antonio Giambruno and Plamen Koshlukov, On the identities of the Grassmann algebras in characteristic $p>0$, Israel J. Math. 122 (2001), 305-316, DOI 10.1007/BF02809905. MR.1826505

[GT09] A. V. Grishin and L. M. Tsybulya, On the T-space and multiplicative structure of a relatively free Grassmann algebra (Russian, with Russian summary), Mat. Sb. 200 (2009), no. 9, 41-80, DOI 10.1070/SM2009v200n09ABEH004038; English transl., Sb. Math. 200 (2009), no. 9-10, 1299-1338. MR2583970

[GTS11] A. V. Grishin, L. M. Tsybulya, and A. A. Shokola, On T-spaces and relations in relatively free Lie nilpotent associative algebras (Russian, with English and Russian summaries), Fundam. Prikl. Mat. 16 (2010), no. 3, 135-148, DOI 10.1007/s10958-011-0515-3; English transl., J. Math. Sci. (N.Y.) 177 (2011), no. 6, 868-877. MR2786534

[GZ05] Antonio Giambruno and Mikhail Zaicev, Polynomial identities and asymptotic methods, Mathematical Surveys and Monographs, vol. 122, American Mathematical Society, Providence, RI, 2005. MR2176105

[Kau18] Aaron Kaufer, Superalgebra in characteristic 2, 2018. arXiv:1804.00824

[Kem91] Aleksandr Robertovich Kemer, Ideals of identities of associative algebras, Translations of Mathematical Monographs, vol. 87, American Mathematical Society, Providence, RI, 1991. Translated from the Russian by C. W. Kohls. MR.1108620

[Kem95] Alexander Kemer, Multilinear identities of the algebras over a field of characteristic $p$, Internat. J. Algebra Comput. 5 (1995), no. 2, 189-197, DOI 10.1142/S0218196795000124. MR.1328550

[KR73] D. Krakowski and A. Regev, The polynomial identities of the Grassmann algebra, Trans. Amer. Math. Soc. 181 (1973), 429-438, DOI 10.2307/1996643. MR325658

[KT94] Issai Kantor and Ivan Trishin, On a concept of determinant in the supercase, Comm. Algebra 22 (1994), no. 10, 3679-3739, DOI 10.1080/00927879408825050. MR.1280094

[LPT05] Joel Louswa, Adilson Eduardo Presto, and Alan Tarr, A new computation of the codimension sequence of the Grassmann algebra, Rose-Hulman Undergraduate Math. J. 6 (2005), no. 2 .

[Tsy09] L. M. Tsybulya, Theorems on equalization and monomiality in a relatively free Grassmann algebra (Russian, with English and Russian summaries), Fundam. Prikl. Mat. 14 (2008), no. 5, 197-218, DOI 10.1007/s10958-009-9714-6; English transl., J. Math. Sci. (N.Y.) 163 (2009), no. 6, 759-773. MR2533589

[Ven16] Siddharth Venkatesh, Hilbert basis theorem and finite generation of invariants in symmetric tensor categories in positive characteristic, Int. Math. Res. Not. IMRN 16 (2016), 5106-5133, DOI 10.1093/imrn/rnv305. MR.3556434

[Zel91] E. I. Zel'manov, Superalgebras and identities, Algebra and analysis (Kemerovo, 1988), Amer. Math. Soc. Transl. Ser. 2, vol. 148, Amer. Math. Soc., Providence, RI, 1991, pp. 39-46, DOI 10.1090/trans2/148/04. MR1109062 
Department of Mathematics, Bar-Ilan University, 52900 Ramat-Gan, Israel Email address: dorgal111@gmail.com

Department of Mathematics, Bar-Ilan University, 52900 Ramat-Gan, Israel; Moscow Institute of Physics and Technology, Institutskiy Pereulok, 9, Dolgoprudny, Moscow OBlast, Russia 141701

Email address: beloval@cs.biu.ac.il

Department of Mathematics, Bar-Ilan University, 52900 Ramat-Gan, Israel

Email address: vishne@math.biu.ac.il 